\title{
lons confined in spherical dielectric cavities modeled by a splitting field-theory
}

Leo Lue ${ }^{1, \text { a) }}$ and Per Linse ${ }^{2}$

${ }^{1)}$ Department of Chemical and Process Engineering, University of Strathclyde

James Weir Building, 75 Montrose Street, Glasgow G1 1XJ,

United Kingdom

${ }^{2)}$ Physical Chemistry, Department of Chemistry

Lund University, P.O. Box 124, S-221 00 Lund, Sweden

(Dated: 13 April 2015)

The properties of ions confined within spherical dielectric cavities are examined by a splitting field-theory and Monte Carlo simulations. Three types of cavities are considered: one possessing a uniform surface charge density, one with a uniform volume charge density, and one containing mobile ions. In all cases, mobile counterions are present within the dielectric sphere. The splitting theory is based on dividing the electrostatic interaction into longand short-wavelength contributions and applying different approximations on the two contributions. The splitting theory works well for the case where the dielectric constant of the confining sphere is equal to or less than that of the medium external to the sphere. Nevertheless, by extending the theory with a virial expansion, the predictions are improved. However, when the dielectric constant of the confining sphere is greater than that of the medium outside the sphere, the splitting theory performs poorly, only qualitatively agreeing with the simulation data. In this case, the strong-coupling expansion does not seem to work well, and a modified mean-field theory where the counterions interact directly with only their own image charge gives improved predictions. The splitting theory works best for the system with a uniform surface charge density and worst for the system with a uniform volume charge density. Increasing the number of ions within the sphere, at a fixed radius, tends to increase the ion density near the surface of the sphere and leads to a depletion region in the sphere interior; however, varying the ion number does not lead to any qualitative changes in the performance of the splitting theory.

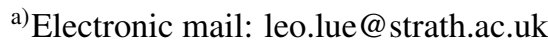




\section{INTRODUCTION}

In many situations, charged species become restricted or confined within a dielectric cavity. We have, e.g., ions in water-in-oil emulsions, ions in particles composed of a charged polymer network, electrolytes in ion exchange membranes, and electrolytes in ion channels. Beside describing the confinement of the charged species, the interaction between the particles mediated by the (confined) charged species is also of large relevance in describing such colloidal systems on a longer length scale. Consequently, there has been a great deal of effort to understand the behavior of confined ions.

The description of charged ionic systems can be taken from two distinct limits, differing in the strength of the electrostatic interaction. In the weak-coupling limit, where the electrostatic interactions are small in comparison to the thermal energy, spatial charge correlations can be largely neglected. Here, mean-field theories, such as the Poisson-Boltzmann theory, work fairly well. Nevertheless, there are situations where charge correlations still are significant, such as in the presence of dielectric inhomogeneities. Correlation corrections can be made to the mean-field theories by using, e.g., loop expansions ${ }^{1,2}$ and variational methods ${ }^{3-7}$. These methods have been used to successfully describe electrolytes near planar ${ }^{3,4,8,9}$, cylindrical ${ }^{5,6,10}$, and spherical ${ }^{3}$ dielectric interfaces. These approximations assume that the fluctuations in the system can be described by Gaussian statistics and, in general, are accurate when these fluctuations are relatively weak.

When the electrostatic interactions between counterions become significant and the spatial correlation among them becomes large, mean-field approaches break down, and other approximation methods are required. A quite successful approach in the limit of very strong electrostatic interactions is the strong-coupling expansion ${ }^{11-13}$. When counterions are strongly correlated and widely separated from each other, due to their mutual repulsions, the system can be treated as an effective one-body problem. In the strong-coupling expansion, the counterions are treated as largely independent from each other. The strong interactions of the counterions with the fixed surface charge density are explicitly accounted for, and the weaker ion-ion interactions are added as corrections ${ }^{12,14}$. For planar geometries with counterions near a fixed surface charge distribution, this approach has been found to work very well.

While the approximation schemes discussed so far work well in either the weak- or strong-coupling limits, most physical systems lie between these two extremes. One approach to develop theories that work in the intermediate regime has been to divide the electrostatic potential into short-wavelength and long-wavelength contributions ${ }^{15-19}$. Treating the long-wavelength interactions with a mean-field approximation and the short-wavelength correlations with a virial expansion leads to a theory that has been found to work well from the weak to the intermediate and to the strong-coupling regimes for planar geometries ${ }^{18-20}$. This splitting theory reduces to a mean-field theory for systems with weak 
electrostatic interactions and to the strong-coupling expansion for systems with very large electrostatic interactions.

In this work, we examine the ability of the splitting theory to predict the properties of ions confined within a spherical cavity by comparing its predictions with results of Monte Carlo (MC) simulations. This is made for ions trapped in cavities with different distributions of neutralizing charges and different dielectric constants. It is unclear how the splitting theory will perform for such systems, since previous studies of confined ions have been for systems where at least one of the dimensions is of infinite extent, such as planes or cylinders. As the charge density of a system increases, the splitting theory approaches the strong-coupling expansion. This expansion is based on an effective one-body approximation, where the ions are well separated, but in the case of spherical confinement this may no longer be the situation, as the counterions cannot indefinitely distance themselves from each other.

The remainder of this paper is organized as follows. In Sec. II, we briefly present the splitting theory and describe aspects of the theory specific to ionic systems confined in spherical, dielectric cavities. Details of the MC simulations are presented in Sec. III. In Sec. IV, we present results for three types of systems that differ in the manner in which the neutralizing charge is distributed. The first type possesses a uniform surface charge density at the dielectric boundary, the second type contains a uniform volume charge distribution within the sphere, and the final case has ions within the sphere. Finally, the conclusions of this work are summarized in Sec. V.

\section{THEORY}

For a model system of ions confined in a dielectric sphere, we here present (i) the Green's function that describes the electrostatic interactions among the ions and between the ions and the dielectric interface and (ii) a splitting field theory, which is able to predict the radial ion density distribution functions. More detailed derivations of the splitting theory can be found in Refs. 18 and 19.

\section{A. Green's function}

Physically, the Green's function $G_{0}\left(\mathbf{r}, \mathbf{r}^{\prime}\right)$ of the Poisson equation represents the potential generated at position $\mathbf{r}$ by a unit point charge located at position $\mathbf{r}^{\prime}$, and it is defined through the equation

$$
-\frac{1}{4 \pi} \nabla \cdot\left[\varepsilon(\mathbf{r}) \nabla G_{0}\left(\mathbf{r}, \mathbf{r}^{\prime}\right)\right]=\delta^{d}\left(\mathbf{r}-\mathbf{r}^{\prime}\right)
$$

where $\varepsilon(\mathbf{r})$ denotes the spatial variation of the dielectric constant, and $d$ the dimensionality of the space.

Formally, the Green's function $G_{0}\left(\mathbf{r}, \mathbf{r}^{\prime}\right)$ can be divided into two terms as

$$
G_{0}\left(\mathbf{r}, \mathbf{r}^{\prime}\right)=G_{\text {hom }}\left(\mathbf{r}, \mathbf{r}^{\prime}\right)+G_{\text {het }}\left(\mathbf{r}, \mathbf{r}^{\prime}\right)
$$


where $G_{\text {hom }}$ is the Green's function of a system with a homogeneous dielectric constant, and $G_{\text {het }}$ is the influence of spatial variations in the dielectric constant.

We now restrict ourselves to a three-dimensional $(d=3)$ space containing a sphere of radius $R$ with a dielectric constant $\varepsilon_{1}$ embedded in a medium with a dielectric constant $\varepsilon_{2}$. In this case, the two terms in Eq. (2) can be expressed as

$$
G_{\text {hom }}\left(\mathbf{r}, \mathbf{r}^{\prime}\right)=\frac{1}{\varepsilon_{1}\left|\mathbf{r}-\mathbf{r}^{\prime}\right|}
$$

and $^{3}$

$$
G_{\text {het }}\left(\mathbf{r}, \mathbf{r}^{\prime}\right)=-\frac{\Delta}{\varepsilon_{1} R} \sum_{l=0}^{\infty} \sum_{m=-l}^{l} \frac{(l+1)}{l+\zeta}\left(\frac{r}{R} \frac{r^{\prime}}{R}\right)^{l} P_{l}(\cos \gamma)
$$

where $\eta=\varepsilon_{1} / \varepsilon_{2}, \Delta=(1-\eta) /(1+\eta), \zeta=(1+\eta)^{-1}, r$ is the distance of $\mathbf{r}$ from the center of the sphere, $r^{\prime}$ is the distance of $\mathbf{r}^{\prime}$ from the center of the sphere, and $\gamma$ is the angle between the vectors $\mathbf{r}$ and $\mathbf{r}^{\prime}$.

Mathematically, Eq. (4) can be re-expressed ${ }^{20,21}$ in a more rapidly converging series. For the case where both $\mathbf{r}$ and $\mathbf{r}^{\prime}$ lie inside the dielectric boundary,

$$
\begin{aligned}
G_{\text {het }}\left(\mathbf{r}, \mathbf{r}^{\prime}\right)=\frac{\eta}{\varepsilon_{1} R} & \frac{1-\eta}{1+\eta}\left\{-\frac{1}{\eta}\left(1-2 t \cos \gamma+t^{2}\right)^{-1 / 2}\right. \\
& +\frac{t^{-1}}{1+\eta} \ln \frac{\left(1-2 t \cos \gamma+t^{2}\right)^{1 / 2}-t+\cos \gamma}{1+\cos \gamma} \\
& \left.-\frac{\eta}{1+\eta}\left[1+\sum_{l=1}^{\infty} \frac{1}{(l+1)} \frac{t^{l} P_{l}(\cos \gamma)}{(1+\eta) l+1}\right]\right\}
\end{aligned}
$$

where $t \equiv r r^{\prime} / R^{2}$.

The surface charge polarization, originating from a point charge inside the dielectric boundary, gives rise to an interaction energy involving the ion that is proportional to $G_{\text {het }}(\mathbf{r}, \mathbf{r})$ and given by

$$
\begin{array}{r}
G_{\text {het }}(\mathbf{r}, \mathbf{r})=\frac{\eta}{\varepsilon_{1} R} \frac{1-\eta}{1+\eta}\left\{-\frac{1}{\eta}|1-t|^{-1}+\frac{t^{-1}}{1+\eta} \ln |1-t|\right. \\
\left.-\frac{\eta}{1+\eta}\left[1+\sum_{l=1}^{\infty} \frac{1}{(l+1)} \frac{t^{l}}{(1+\eta) l+1}\right]\right\}
\end{array}
$$

When $\eta<1$ and/or both cations and anions are present, the ions possess a nonzero radius to avoid an infinite negative Coulomb energy; otherwise point ions are employed to limit the number of parameters.

\section{B. Splitting theory}

The basic idea of the splitting theory is to separate the electrostatic interactions into longwavelength (large-distance) and short-wavelength (short-distance) contributions and to evaluate each 
of these within an appropriate approximation scheme ${ }^{15-17,22,23}$. This division is achieved by splitting the Green's function $G_{0}\left(\mathbf{r}, \mathbf{r}^{\prime}\right)$ of the Poisson equation as

$$
G_{0}\left(\mathbf{r}, \mathbf{r}^{\prime}\right)=G_{s}\left(\mathbf{r}, \mathbf{r}^{\prime}\right)+G_{l}\left(\mathbf{r}, \mathbf{r}^{\prime}\right)
$$

where $G_{l}=\mathcal{P} G_{0}$ is the long-wavelength portion of the Green's function, $G_{s}=(1-\mathcal{P}) G_{0}$ is the shortwavelength portion of the Green's function, and $\mathcal{P}$ is an operator that filters out short-wavelength contributions.

In this work, we choose $\mathcal{P}=\left[1-\sigma^{2} \nabla^{2}+\sigma^{4} \nabla^{4}\right]^{-1}$, where $\sigma$ is the length scale that separates shortwavelength and long-wavelength phenomena. For this choice of operator $\mathcal{P}$, the short-wavelength Green's function $G_{s}$ becomes

$$
G_{s}\left(\mathbf{r}, \mathbf{r}^{\prime}\right)=G_{\mathrm{hom}, \mathrm{s}}\left(\mathbf{r}, \mathbf{r}^{\prime}\right)+G_{\mathrm{het}, \mathrm{s}}\left(\mathbf{r}, \mathbf{r}^{\prime}\right)
$$

where

$$
G_{\mathrm{hom}, \mathrm{s}}\left(\mathbf{r}, \mathbf{r}^{\prime}\right)=\frac{e^{-\frac{\sqrt{3}\left|\mathbf{r}-\mathbf{r}^{\prime}\right|}{2 \sigma}}}{\varepsilon_{1}\left|\mathbf{r}-\mathbf{r}^{\prime}\right|}\left[\cos \frac{\left|\mathbf{r}-\mathbf{r}^{\prime}\right|}{2 \sigma}+\frac{1}{\sqrt{3}} \sin \frac{\left|\mathbf{r}-\mathbf{r}^{\prime}\right|}{2 \sigma}\right]
$$

and

$$
G_{\text {het, } \mathrm{s}}\left(\mathbf{r}, \mathbf{r}^{\prime}\right)=-\frac{2}{\pi} \frac{\Delta}{\varepsilon_{1}} \sum_{l=0}^{\infty} \frac{l+1}{l+\zeta}\left(\frac{r^{\prime}}{R}\right)^{l}(2 l+1) \frac{2}{\sqrt{3} \sigma} \operatorname{Im}\left[e^{i \pi / 6} i_{l}(\operatorname{ar}) k_{l}(a R)\right] P_{l}(\cos \gamma),
$$

where $a^{-1}=\sigma e^{i \pi / 6}, i_{l}$ is a modified spherical Bessel function of the first kind, and $k_{l}$ is a modified spherical Bessel function of the second kind.

If the contributions to the partition function due to the short-wavelength and long-wavelength fluctuations in the system are determined exactly, then the resulting free energy (and other properties) of the system will be independent of the choice of the operator $\mathcal{P}$. However, in practice, approximations must be made to evaluate these terms, and, as a consequence, the free energy developed by the theory will be dependent on the precise manner in which the Green's function is split, as well as the nature of the approximations.

This is similar to the use of the Ewald summation method to evaluate electrostatic interactions. In this case, the short-range terms are summed directly, while the long-range terms are summed in Fourier space. Without dividing the interaction, the resulting calculations are impractically slow; however, with the splitting, both the short-range and long-range terms converge much more rapidly than the original series.

In this work, the short-wavelength contribution is approximated by a cumulant expansion truncated at second order, which corresponds to using the second virial approximation for the short-wavelength contributions. The long-wavelength contributions are treated using a mean-field approximation. With 
these approximations, the free energy functional $F$ of the system is given by ${ }^{18,19,24}$

$$
\begin{aligned}
& \beta F[\rho]=\sum_{\alpha} \int d \mathbf{r} \rho_{\alpha}(\mathbf{r})\left[\ln \rho_{\alpha}(\mathbf{r}) \Lambda_{\alpha}^{3}-1+\beta u_{\alpha}(\mathbf{r})\right]-\frac{1}{2} \sum_{\alpha \alpha^{\prime}} \int d \mathbf{r} d \mathbf{r}^{\prime} \rho_{\alpha}(\mathbf{r}) f_{\alpha \alpha^{\prime}}\left(\mathbf{r}, \mathbf{r}^{\prime}\right) \rho_{\alpha^{\prime}}\left(\mathbf{r}^{\prime}\right) \\
& \quad+\beta E_{\mathrm{se}}-\frac{1}{2 \beta} \int d \mathbf{r} d \mathbf{r}^{\prime} \bar{\psi}_{l}(\mathbf{r}) G_{0}^{-1}\left(\mathbf{r}, \mathbf{r}^{\prime}\right) \bar{\psi}_{l}\left(\mathbf{r}^{\prime}\right)+\int d \mathbf{r}\left[\Sigma(\mathbf{r})+\sum_{\alpha} q_{\alpha} \rho_{\alpha}(\mathbf{r})\right] i \bar{\psi}_{l}(\mathbf{r})+\cdots
\end{aligned}
$$

where $\rho_{\alpha}(\mathbf{r})$ is the number density, $q_{\alpha}$ is the charge, $\Lambda_{\alpha}$ is the thermal wavelength of ions of type $\alpha$, and the ellipses represent higher order virial corrections and cumulant terms not included in the present theory.

In Eq. (11), $u_{\alpha}(\mathbf{r})$ is an effective one-body potential acting on each ion of type $\alpha$ and is given by

$$
u_{\alpha}(\mathbf{r})=q_{\alpha} \int d \mathbf{r}^{\prime} G_{s}\left(\mathbf{r}, \mathbf{r}^{\prime}\right) \Sigma\left(\mathbf{r}^{\prime}\right)+\frac{q_{\alpha}^{2}}{2} G_{\mathrm{het}}(\mathbf{r}, \mathbf{r})-\frac{q_{\alpha}^{2}}{2} \mathcal{P} G_{\mathrm{hom}}(\mathbf{r}, \mathbf{r})+u_{\alpha}^{\mathrm{ext}}(\mathbf{r}) .
$$

where $u_{\alpha}^{\text {ext }}(\mathbf{r})$ is an external, non-electrostatic interaction imposed on ions of type $\alpha$. The term $E_{\mathrm{se}}$ is the short-wavelength self energy of the fixed charge density $\Sigma(\mathbf{r})$, which is given by

$$
E_{\mathrm{se}}=\frac{1}{2} \int d \mathbf{r} d \mathbf{r}^{\prime} \Sigma(\mathbf{r}) G_{s}\left(\mathbf{r}, \mathbf{r}^{\prime}\right) \Sigma\left(\mathbf{r}^{\prime}\right)
$$

The quantity $\bar{\psi}_{l}(\mathbf{r})$ is the long-range portion of the mean electrostatic potential:

$$
\bar{\psi}_{l}=\mathcal{P} \beta \phi
$$

where $\phi$ is the mean electrostatic potential, which is obtained from solving the Poisson equation

$$
-\frac{1}{4 \pi} \nabla \cdot[\varepsilon(\mathbf{r}) \nabla \phi(\mathbf{r})]=\sum_{\alpha} q_{\alpha} \rho_{\alpha}(\mathbf{r})+\Sigma(\mathbf{r}) .
$$

Finally, the Mayer $f$-function, which appears in Eq. (11), is given by

$$
f_{\alpha \alpha^{\prime}}\left(\mathbf{r}, \mathbf{r}^{\prime}\right)=e^{-\beta q_{\alpha} q_{\alpha^{\prime}} G_{s}\left(\mathbf{r}, \mathbf{r}^{\prime}\right)}-1
$$

The theory with $f_{\alpha \alpha^{\prime}}\left(\mathbf{r}, \mathbf{r}^{\prime}\right)=0$ will be referred to as the splitting theory and with $f_{\alpha \alpha^{\prime}}\left(\mathbf{r}, \mathbf{r}^{\prime}\right)$ given by Eq. (16) to as the splitting theory with virial correction.

The physics of electrolyte systems near a fixed charge density has been described through two different limiting situations ${ }^{12,13}$. In the limit of low fixed charge density or small counterion valency, the counterions are fairly uncorrelated and the Poisson-Boltzmann theory works well. When the fixed charge density or the counterion valency is large, then the physics is dominated by electrostatic interactions between the counterions. In this situation, the mutual repulsion between counterions leads to a large correlation hole surrounding each of the counterions. The strong-coupling expansion accounts for this by considering the interaction with the fixed charge density, and then with other ions. 
However, in the case when the ions are restricted to a confined space, then the size of the correlation hole is limited and the interactions between ions become more significant. It is unclear how the strong-coupling expansion performs in this situation.

While the virial expansion does yield the proper leading-order behavior in the strong-coupling limit $^{12}$, it does not give the correct form for the leading order correction ${ }^{14,19,25,26}$, which was first determined numerically by Moreira and $\mathrm{Netz}^{25}$ for the planar geometry. For example, the leadingorder correction for the pressure between two charged plates with intervening counterions varies inversely as the square root of the coupling parameter, while the virial expansion yields a correction that varies inversely with the coupling parameter. However, the dependence of the parameter $\sigma$ within the splitting theory allows it to give the correct prediction of the power law, but changing the level of approximation of the integration of the short-range fluctuations will change the precise value of the proportionality constant. Consequently, it is able to give ${ }^{24}$ an excellent description of the pressure in the two plate geometry from the strong- to weak- coupling limits.

The splitting theory is able to pass between these two limiting cases. The value of the splitting parameter is determined by making the free energy stationary with respect to variations in $\sigma$ :

$$
\frac{\partial F}{\partial \sigma}=0
$$

In the limit $\sigma \rightarrow 0$, the theory reduces to a modified form of the Poisson-Boltzmann theory, where the ions interact explicitly with their image charges. When $\sigma \rightarrow \infty$, the theory becomes related to the strong-coupling expansion ${ }^{11,12}$. The "optimal" value of the splitting parameter will depend on the precise approximation used to evaluate the free energy. Hence, the particular values determined for $\sigma$ will differ between the splitting theory with and without the virial correction.

Once the optimal value of the splitting parameter is known, all the static properties of the system can be determined from the free energy functional given in Eq. (11). The chemical potential of an ion of type $\alpha$ in the system is given by

$$
\gamma_{\alpha}(\mathbf{r})=\ln \rho_{\alpha}(\mathbf{r}) \Lambda_{\alpha}^{d}+\beta u_{\alpha}(\mathbf{r})+q_{\alpha} i \bar{\psi}_{l}(\mathbf{r})-\sum_{\alpha^{\prime}} \int d \mathbf{r}^{\prime} f_{\alpha \alpha^{\prime}}\left(\mathbf{r}, \mathbf{r}^{\prime}\right) \rho_{\alpha^{\prime}}\left(\mathbf{r}^{\prime}\right)+\cdots
$$

The density distribution of ions of type $\alpha$ can be obtained by solving the above equation:

$$
\rho_{\alpha}(\mathbf{r})=\Lambda_{\alpha}^{-d} e^{\gamma_{\alpha}(\mathbf{r})-\beta u_{\alpha}(\mathbf{r})-q_{\alpha} i \bar{\psi}_{l}(\mathbf{r})+\sum_{\alpha^{\prime}} \int d \mathbf{r}^{\prime} f_{\alpha \alpha^{\prime}}\left(\mathbf{r}, \mathbf{r}^{\prime}\right) \rho_{\alpha^{\prime}}\left(\mathbf{r}^{\prime}\right)+\cdots}
$$

At equilibrium, the chemical potential $\gamma_{\alpha}(\mathbf{r})$ of each type of ion $\alpha$ should be constant throughout the system.

For a given choice of $\sigma$, Eqs. (19), (15), and (14) are solved numerically to obtain the ion density profile and electrostatic potential. In this work, the chemical potential is spatially uniform, and its value is adjusted so that the total charge of the counterions neutralizes the surface charge. From the 
ion density profile, the charge density follows, and the Poisson equation, Eq. (15), is solved using the finite difference method, while the link between the long-range portion of the mean electrostatic potential $i \bar{\psi}_{l}$ and the mean electrostatic potential $\phi$ is evaluated using the fast Fourier transfer method. This then leads to a new estimate of the ion density profile. This process is repeated until the solution has converged. The splitting parameter $\sigma$ is then varied until the free energy functional, given in Eq. (11), is maximized.

\section{SIMULATION DETAILS}

Monte Carlo (MC) simulations were performed in the canonical ensemble, (i.e. at constant number of particles, volume, and temperature) for systems of ions confined in a dielectric cavity. The electrostatic energy originating from $G_{\text {het }}\left(\mathbf{r}, \mathbf{r}^{\prime}\right)$ (referred to as the polarization energy) was evaluated using Eq. (5). This expression contains a term that involves an infinite sum over $l$, which needs to be truncated for it to be evaluated numerically. Fortunately, this sum converges extremely rapidly. In no case were significant differences observed between truncating the sum after one term and two terms. The data presented are calculated by approximating the sum with only one term.

After equilibration, each simulation involved $10^{7} \mathrm{MC}$ trial moves per ion for systems containing $N=5$ ions and $10^{6} \mathrm{MC}$ trial moves for $N=100$ ions. The value of trial displacements ranged from 2 to $10 \AA$. Radial ion distributions were determined by using a histogram width of $0.2 \AA$. Statistical uncertainties were calculated using block averaging by subdividing each simulation into ten equally sized blocks. The integrated MC/molecular dynamics/Brownian dynamics simulation package MOLSIM for molecular systems was employed ${ }^{27}$. Both the ion polarization self energy and the ion-ion polarization interactions were included in the MC simulations. The inclusion of the polarization energy lengthened the MC simulation times by a factor of 2.5, as compared to the time where the polarization energy evaluation was switched off.

\section{RESULTS AND DISCUSSION}

We now apply the theory developed in Sec. II to a system of ions possessing a charge of magnitude $q$ and hard-sphere radius $R_{\text {ion }}$. Beside the Coulomb interaction, the interaction between ions also possesses a short-range, excluded volume contribution $u^{\text {short }}(r)$ given by

$$
u^{\text {short }}(r)=\left\{\begin{array}{l}
\infty \text { for } 0<r<2 R_{\text {ion }} \\
0 \text { for } 2 R_{\text {ion }}<r
\end{array} .\right.
$$


The ions are confined within a sphere of radius $R$ though an external potential $u^{\text {ext }}(r)$ given by

$$
u^{\mathrm{ext}}(r)=\left\{\begin{array}{l}
0 \quad \text { for } 0<r<R-R_{\text {ion }} \\
\infty \text { for } R-R_{\text {ion }}<r
\end{array} .\right.
$$

The dielectric constant of the medium inside the confining sphere is $\varepsilon_{1}$, and the dielectric constant of the medium external to the confining sphere is $\varepsilon_{2}$. Consequently, the dielectric constant of the system $\varepsilon(\mathbf{r})$ varies as:

$$
\varepsilon(r)=\left\{\begin{array}{l}
\varepsilon_{1} \text { for } 0<r<R \\
\varepsilon_{2} \text { for } R<r
\end{array} .\right.
$$

We consider three different types of such systems, which are schematically depicted in Fig. 1. The first system is comprised of a set of identical ions, which is neutralized by a uniform surface charge density on the sphere [Fig. 1(a)]. The second system consists of a set of same ions, which is neutralized by a uniform volume charge density within the sphere [Fig. 1(b)]. The final system is a simple electrolyte, with both ions and counterions, confined within a dielectric sphere [Fig. 1(c)].

\section{A. Counterions in a dielectric cavity with a uniform surface charge density}

We first consider a system of $N$ ions with charge $q$ and hard-sphere radius $R_{\text {ion }}$, which are confined within the dielectric sphere. The uniform and spherical surface charge density $\Sigma(\mathbf{r})$ is located at the dielectric discontinuity and is given by

$$
\Sigma(\mathbf{r})=\frac{Q}{4 \pi R^{2}} \delta(r-R)
$$

where $Q=-N q$ is the total charge of the spherical shell. This fixed charge density neutralizes the charges of the ions. This system is depicted in Fig. 1(a).

The electrostatic potential $\phi(\mathbf{r})$ generated by a charge distribution $\Sigma(\mathbf{r})$ is given by

$$
\phi(\mathbf{r})=\int d \mathbf{r}^{\prime} G_{0}\left(\mathbf{r}, \mathbf{r}^{\prime}\right) \Sigma\left(\mathbf{r}^{\prime}\right)
$$

which for a uniform and spherical surface charge density reduces to

$$
\phi(\mathbf{r})=\frac{Q}{\varepsilon_{2} R}\left\{\begin{array}{ll}
1 & \text { for } r<R, \\
R / r & \text { for } R<r
\end{array} .\right.
$$

Outside the sphere, the electrostatic potential decays inversely with the distance from the center of the sphere. Inside the sphere, the electrostatic potential from the fixed and uniform charge density is constant; therefore, an ion within the sphere will feel no force from the surface charge density, regardless of its position. 
The short-wavelength component of the electrostatic potential generated by a fixed charge density is given by

$$
\phi_{s}(\mathbf{r})=\int d \mathbf{r}^{\prime} G_{s}\left(\mathbf{r}, \mathbf{r}^{\prime}\right) \Sigma\left(\mathbf{r}^{\prime}\right)
$$

This quantity is important to the theory, as it contributes to the effective one-body interaction potential that an ion experiences. For a uniform and fixed spherical surface charge density, this becomes

$$
\phi_{s}(\mathbf{r})=\frac{Q}{\sqrt{3} \varepsilon_{2} R} f_{2}(r) .
$$

where

$$
f_{2}(r)=\frac{\sigma}{r}\left[e^{-\frac{\sqrt{3}(R-r)}{2 \sigma}} \cos \left(\frac{R-r}{2 \sigma}\right)-e^{-\frac{\sqrt{3}(R+r)}{2 \sigma}} \cos \left(\frac{R+r}{2 \sigma}\right)\right] .
$$

The difference $f_{2}(r)-f_{2}(0)$ is shown in Fig. 2 for different values of the splitting parameter $\sigma$. When $\sigma$ is much larger than the radius of the confining sphere $R$, the short-range potential $\phi_{s}(\mathbf{r})$ is constant, as expected. As $\sigma / R$ decreases, $\phi_{s}(\mathbf{r})$ becomes larger at the surface of the sphere until $\sigma / R \approx 0.2$. Thereafter, the potential at the surface begins to decrease with decreasing $\sigma / R$. For $\sigma / R \lesssim 0.2$, the potential is constant except for a region within a distance $\approx \sigma / R$ from the surface of the sphere.

For this system, the one-body potential $u(\mathbf{r})$ is given by

$$
\beta u(r)=\frac{\eta Q / q}{\sqrt{3}} \frac{l_{B}}{R} f_{2}(r)+\frac{\beta q^{2}}{2} G_{\text {het }}(\mathbf{r}, \mathbf{r})-\frac{l_{B}}{2 \sqrt{3} \sigma}+\beta u^{\mathrm{ext}}(r) .
$$

where $l_{B}=\beta q^{2} / \varepsilon_{1}$ is the Bjerrum length. In the limit $\sigma / R \rightarrow 0$, the short-wavelength interaction of the ions with the uniform surface charge density vanishes. When $\sigma / R \gg 1$, the one-body potential becomes

$$
\beta u(r) \approx \eta(Q / q) \frac{l_{B}}{R}+\frac{\beta q^{2}}{2} G_{\text {het }}(\mathbf{r}, \mathbf{r})+\beta u^{\mathrm{ext}}(r)
$$

The short-wavelength self energy of the fixed and uniform surface charge density is given by

$$
E_{\mathrm{se}}=\frac{Q^{2}}{2 \sqrt{3} \varepsilon_{2} R} \frac{\sigma}{R}\left[1-e^{-\sqrt{3} R / \sigma} \cos \left(\frac{R}{\sigma}\right)\right]
$$

In the limit $\sigma / R \rightarrow \infty$, the self energy of the fixed charge becomes

$$
E_{\mathrm{se}} \approx \frac{Q^{2}}{2 \varepsilon_{2} R}
$$

which is the self energy of a spherical shell charge.

As mentioned previously, the value of the splitting parameter $\sigma$ is chosen such that the free energy is stationary with respect to $\sigma$ [see Eq. (17)]. The variation of the optimal value of $\sigma$ with the number of ions $N$ in the system is shown in Fig. 3. For the case $N=1$, the splitting parameter diverges (i.e. $\sigma \rightarrow \infty$ ), and the theory reduces to that where the ion simply interacts with the fixed charge density. As the number of ions in the cavity increases, the screening effect increases and $\sigma$ decreases. For 
sufficiently large numbers of ions in the sphere, the splitting parameter varies as $\sigma / R \propto N^{-1 / 2}$ for $\eta=1$ and 0.1 . This corresponds to $\sigma$ being proportional to the spacing between ions when they are packed near the surface of the confining sphere.

For a fixed, and not too small, number of ions in the system, $\sigma / R$ increases with the radius of the confining sphere. The increase is largest for large $\eta$ and vanishes for $\eta=0.1$. The strength of the self energy of the fixed charge and the one-body potential is proportional to $1 / R$ [see Eqs. (28) and (27)]. Therefore, we expect that as the radius of the confining sphere becomes larger, the contribution of these terms will become smaller, and the mean-field limit will prevail. As the radius of the confining sphere shrinks and $\sigma / l_{B}$ becomes smaller, we find that the one-body term begins to dominate the distribution of the ions.

Density profiles for $N=5$ point ions confined within a sphere at $\eta=1$ (no dielectric interface) are shown in Fig. 4(a) and for $N=100$ ions in Fig. 4(b) at sphere radii $R / l_{B}=1$ and 10 . In all cases, the density maxima appear at the surface of the confining sphere, which originates from the repulsive ion-ion interaction. Remember that the uniform surface charge density does not impose any force on the ions. For this case, the theory reproduces well the MC simulation data, though it slightly underestimates the ion density at the surface and slightly overestimates the density at the center of the sphere. The addition of the virial correction improves the theory from the original splitting theory for $N=5$.

The corresponding density profiles for point ions at $\eta=10$ (the dielectric constant of the confining sphere is greater than that of its surroundings) are shown in Fig. 5. Here, the ion density is zero at the surface of the sphere, and density maxima occur within the interior of the dielectric sphere. The inward shift of the density maxima is due to induced surface charges that repel the ions from the dielectric interface. As the number of ions in the sphere increases, the density maxima are pushed closer to the surface of the sphere, decreasing the ion concentration in the center of the sphere and resulting in a fairly high and sharp peak. For these systems, the prediction of the splitting theory is poor. Unlike the case $\eta=1$, the virial correction actually worsens the predictions of the splitting theory. Adding short-range correlations to this order is insufficient to quantitatively describe the system. This suggests that using the cumulant (virial) expansion to approximate the short-wavelength fluctuations may not be best choice. Perhaps another approximation scheme, such as a loop expansion, could lead to improved results.

Interestingly, setting $\sigma=0$ in the splitting theory (both with or without the virial correction) yields better predictions for the ion density profiles. This corresponds to the case where the ions interact only with their own image charge in the one-body potential, and the interaction of the ions with the fixed charge density (which is zero in this case) and the other ions is treated within a meanfield approximation. Physically, this corresponds to the situation where the ions are relatively "fixed" 
and, consequently, the electrostatic potential does not significantly fluctuate. This limit of the splitting theory is quite similar to the approach developed in Ref. 28, where the Poisson-Boltzmann equation was modified to explicitly incorporate the interaction between ions and their image charges.

Finally, density profiles for ions at $\eta=0.1$ (the dielectric constant of the confining sphere is smaller than that of its exterior surroundings) are shown in Fig. 6. Here, the ions are attracted by the dielectric discontinuity, and a hard-sphere radius $R_{\text {ion }}=0.05 R$ has been assigned to the ions to avoid the divergence of the attractive polarization energy at the dielectric discontinuity [see Eq. (21)]. As for $\eta=1$, the splitting theory performs well, and the performance is (i) better for $N=5$ than for $N=100$ ions and (ii) better with virial correction included than excluded.

\section{B. Counterions in a dielectric cavity with a uniform volume charge density}

Again consider a system of $N$ ions, each possessing the charge $q$ and hard-sphere radius $R_{\text {ion, }}$, which are confined within a dielectric sphere; however, now there is a uniform spherical volume charge density $\Sigma(\mathbf{r})$ present inside the dielectric sphere, which is given by

$$
\Sigma(\mathbf{r})=\frac{3 Q}{4 \pi R^{3}}\left\{\begin{array}{l}
1 \text { for } r<R \\
0 \text { for } R<r
\end{array}\right.
$$

where $r$ is the distance from the center of the sphere, and $Q=-N q$ is the total charge of the volume charge density. The volume charge density serves as a neutralizing background to the ions. This system is depicted schematically in Fig. 1(b).

The electrostatic potential $\phi(\mathbf{r})$ generated by this uniform volume charge density is

$$
\phi(\mathbf{r})=\frac{Q}{\varepsilon_{1} R} \begin{cases}\frac{1}{2}\left(3-\frac{r^{2}}{R^{2}}\right)-(1-\eta) & \text { for } r<R \\ \frac{\eta R}{r} & \text { for } R<r\end{cases}
$$

This acts as a harmonic potential that draws the ions toward the center of the sphere with a linear force. In this case, the short-wavelength potential generated by the fixed charge is

$$
\phi_{s}(r)=\frac{Q}{2 \varepsilon_{1} R} f_{1}(r)-(1-\eta) \frac{Q}{\sqrt{3} \varepsilon_{1} R} f_{2}(r)
$$

where

$$
\begin{aligned}
f_{1}(r)=\frac{\sigma^{2}}{R^{2}} & \left\{6+\left(\sqrt{3}+3 \frac{\sigma}{R}\right) \frac{R}{r}\left[e^{-\frac{\sqrt{3}(R-r)}{2 \sigma}} \sin \left(\frac{R-r}{2 \sigma}\right)-e^{-\frac{\sqrt{3}(R+r)}{2 \sigma}} \sin \left(\frac{R+r}{2 \sigma}\right)\right]\right. \\
& \left.-\left(3+\sqrt{3} \frac{\sigma}{R}\right) \frac{R}{r}\left[e^{-\frac{\sqrt{3}(R-r)}{2 \sigma}} \cos \left(\frac{R-r}{2 \sigma}\right)-e^{-\frac{\sqrt{3}(R+r)}{2 \sigma}} \cos \left(\frac{R+r}{2 \sigma}\right)\right]\right\} .
\end{aligned}
$$

The ratio $f_{1}(r) / f_{1}(0)$ is plotted in Fig. 7 for different values of the splitting parameter. The form of the function is dictated by the ratio $\sigma / R$. The strength of the potential is given by the ratio $R / l_{B}$. 
The smaller the value of $R / l_{B}$, the stronger the short-ranged potential generated by the uniform fixed charge density.

The one-body potential felt by the ions is given by

$$
\beta u(r)=\frac{Q / q}{2} \frac{l_{B}}{R} f_{1}(r)+(1-\eta) \frac{Q / q}{\sqrt{3}} \frac{l_{B}}{R} f_{2}(r)+\frac{\beta q^{2}}{2} G_{\text {het }}(\mathbf{r}, \mathbf{r})-\frac{l_{B}}{2 \sqrt{3} \sigma}+\beta u^{\mathrm{ext}}(r) .
$$

In the limit $\sigma / R \rightarrow 0$, the short-wavelength interaction of the ions with the fixed charge density vanishes. When $\sigma / R \gg 1$, this becomes

$$
\begin{gathered}
\beta u(r) \approx \frac{\beta q Q}{2 \varepsilon_{1} R}\left(3-\frac{r^{2}}{R^{2}}\right)-(1-\eta) \frac{\beta q Q}{\varepsilon_{1} R} \\
+\frac{\beta q^{2}}{2} G_{\text {het }}(\mathbf{r}, \mathbf{r})+\beta u^{\text {ext }}(r) .
\end{gathered}
$$

The corresponding expression for the short-wavelength self-energy of the fixed charge density is

$$
\begin{aligned}
E_{\mathrm{se}}=\frac{3 Q^{2}}{2 \varepsilon_{1} R} \frac{\sigma^{2}}{R^{2}} & {\left[1-\frac{\sqrt{3}}{2} \frac{\sigma}{R}\left(1+\frac{\sigma^{2}}{R^{2}}\right)-\frac{\sqrt{3}}{2} \frac{\sigma}{R}\left(1-\frac{\sigma^{2}}{R^{2}}\right) e^{-\sqrt{3} R / \sigma} \cos \left(\frac{R}{\sigma}\right)\right.} \\
+ & \left.\frac{\sqrt{3}}{2} \frac{\sigma}{R}\left(1+\sqrt{3} \frac{\sigma}{R}\right)\left(\sqrt{3}+\frac{\sigma}{R}\right) e^{-\sqrt{3} R / \sigma} \sin \left(\frac{R}{\sigma}\right)\right] \\
+(1-\eta) & \frac{\sqrt{3} Q^{2}}{4 \varepsilon_{1} R} \frac{\sigma^{2}}{R^{2}}\left[\frac{\sigma}{R}-\sqrt{3}-\left(\sqrt{3}+\frac{\sigma}{R}\right) e^{-\sqrt{3} R / \sigma} \cos \left(\frac{R}{\sigma}\right)\right. \\
+ & \left.\left(1+\sqrt{3} \frac{\sigma}{R}\right) e^{-\sqrt{3} R / \sigma} \sin \left(\frac{R}{\sigma}\right)\right]
\end{aligned}
$$

This is a monotonically increasing function of $\sigma / R$. In the limit $\sigma / R \rightarrow 0$, the self-energy vanishes. In the limit $\sigma / R \rightarrow \infty$, the short-wavelength self-energy of the fixed charge density becomes

$$
E_{\mathrm{se}} \approx \frac{3 Q^{2}}{5 \varepsilon_{1} R}-(1-\eta) \frac{Q^{2}}{2 \varepsilon_{1} R}
$$

which is the bare self-energy of a uniform spherical charge density.

The optimal values of the splitting parameter as a function of the number of ions in the spherical cavity is shown in Fig. 8. For the cases $\eta=1$ and 0.1 , the general dependence of $\sigma / R$ on $N$ is quite similar to that found in the systems with the uniform surface charge density (see Fig. 3), although the $N$-dependence is weaker. However, the results for $\eta=10$ differ substantially from that previously found and are shifted to smaller values of $\sigma / R$. In addition, for $R / l_{B}=0.5$ and 1 there is a critical value of $N$ above which no optimal value of $\sigma$ can be found. This is depicted by the point (see circle in Fig. 8) where the line splits into two branches. In the region between the two branches, characterized by different values of $\sigma / \mathrm{R}$, the splitting theory with the virial correction has no solution.

Figure 9(a) provides ion density profiles for $N=5$ point ions, and Fig. 9(b) for $N=100$ point ions confined in the sphere at $\eta=1$. Again density maxima appear in the interior of the dielectric 
sphere (with a few exceptions). However, here the two balancing forces, giving rise to the density maxima, are (i) the ion-ion repulsion and (ii) the linear inward force from the uniform volume charge density, represented by Eq. (31). Moreover, (i) the location of the density maxima is shifted to a larger radial distance, and the height of the maxima increases with the larger number of ions (larger $N$ ) and (ii) the height of the maxima increases with decreasing system size ( $\operatorname{smaller} R / l_{B}$ ).

While the splitting theory performs adequately for the larger value of $R / l_{B}$, its predictions become increasingly poor as size of the confining sphere decreases. The virial correction improves the splitting theory; however, discrepancies remain, pointing out the importance of higher order virial terms, and their incorporation should lead to an improvement of the theory. Generally, the theory is less poor for the system with the larger number of ions. Finally, the Poisson-Boltzmann theory, which corresponds to the limit $\sigma=0$ of the splitting theory, predicts a uniform ion density profile for this system, independently of the values of $R$ and $N$.

Next, ion density profiles for $N=5$ and $N=100$ point ions confined in the sphere at $\eta=10$ are provided in Fig. 10. In the same manner as for the case with a uniform surface charge density, at $\eta=10$ (i) the polarization of the dielectric interface repels the ions from the sphere surface and all ion densities become zero at the dielectric interface, where the ion-surface polarization energy becomes infinite and (ii) the radial structuring of the ions is enhanced, as compared to $\eta=1$. From the results of the MC simulations, we deduce that the magnitude of the highest peak located closest to the spherical boundary increases with reducing radius of the sphere and is shifted toward the surface with increasing number of ions, whereas the heights of the maxima are basically insensitive to the number of ions.

For the case $R / l_{B}=1$ with $N=100$ ions, the splitting theory with the virial correction has a region with no solution. For these systems, we take the value of $\sigma$ that has the highest free energy, which corresponds to the lower branch of the curve for the splitting parameter (see Fig. 8). As for the case with a uniform surface charge density at $\eta=10$, the splitting theory performs poorly, in general. We note that (i) the location of maxima is too close to the surface of the confining sphere and (ii) the amplitude is too small for $N=5$ and too high for $N=100$ ions. Inclusion of the virial correction improves the amplitude and location of the main peak but leads to a depletion of ions in the center of the sphere, which does not appear in the simulations. Finally, as with the uniform surface charge density, we find that setting $\sigma=0$ in the splitting theory leads to improved predictions.

Figure 11 provides ion density profiles of the confined ions at $\eta=0.1$. Here the ions are subjected to three different types of forces: (i) repulsive ion-ion forces pushing the ions radially outward, (ii) a linear force from the uniform volume charge density pulling ions toward the center of the sphere, and (iii) a force from the surface polarization pulling the ions toward the dielectric discontinuity. As the last force diverges at the discontinuity, we again set $R_{\text {ion }}=0.05 R$ to avoid this divergence. In 
comparison with the case of a uniform surface charge density (Fig. 6), we infer from the results of the MC simulations that (i) accumulation of the ions near the surface is somewhat reduced and (ii) the depletion of the ions in the center of the sphere has almost vanished for $N=5$ and completely vanished for $N=100$.

For $N=5$, the splitting theory without the virial correction overestimates the ion density near the center of the sphere, while with the virial correction it performs reasonably well. The magnitude of the virial correction is significant. For $N=100$, the splitting theory reproduces qualitatively the simulation results, but exaggerates the density minimum next to the global maximum and predicts a secondary density maximum. The virial correction offers a significant improvement in the prediction of the splitting theory.

\section{Simple electrolyte in a dielectric cavity}

Finally, we examine systems having $N$ cations with charge $+q$ and $N$ anions with charge $-q$ confined within the dielectric sphere. The ions have a hard-sphere radius $R_{\text {ion }}=0.5 l_{B}$ [see Eq. (20)] to prevent the Coulomb interaction between cations and anions from diverging [i.e. the cation-anion Mayer $f$-function, Eq. (16), in the free energy functional, Eq. (11)], as well as to prevent the divergence between ions at the dielectric discontinuity when $\eta=0.1$ [see Eq. (21)]. A schematic drawing of these systems is provided in Fig. 1(c).

Monte Carlo simulations for charged hard-sphere systems confined inside a cavity with a dielectric constant much larger than its exterior surroundings have been previously performed ${ }^{29}$ in order to determine the air-water interfacial tension of electrolyte systems. These have been found to be in good agreement with experimental measurements. Note, however, the degree of confinement and the strength of the electrostatic coupling in that study are less extreme than examined here.

Since no fixed charge density is present (i.e. $\Sigma(\mathbf{r})=0$ ), $E_{\mathrm{se}}=0$. The one-body potential is simply given by the image charge generated by the dielectric interface:

$$
\beta u_{ \pm}(r)=\frac{\beta q^{2}}{2} G_{\text {het }}(\mathbf{r}, \mathbf{r})-\frac{l_{B}}{2 \sqrt{3} \sigma}+\beta u^{\text {ext }}(r)
$$

Because the cations and anions are symmetric, there is no charge separation in the system, i.e., the local charge density is everywhere zero. In addition, the average electric potential is constant throughout the system, so we take $\bar{\psi}_{l}=0$.

In this case, the free energy $F$ of the system simplifies to

$$
\begin{aligned}
\beta F[\rho]=2 & \int d \mathbf{r} \rho_{ \pm}(\mathbf{r})\left[\ln \rho_{ \pm}(\mathbf{r}) \Lambda_{ \pm}^{d}-1+\beta u_{ \pm}(\mathbf{r})\right] \\
& -\int d \mathbf{r} d \mathbf{r}^{\prime} \rho_{ \pm}(\mathbf{r})\left[f_{++}\left(\mathbf{r}, \mathbf{r}^{\prime}\right)+f_{+-}\left(\mathbf{r}, \mathbf{r}^{\prime}\right)\right] \rho_{ \pm}\left(\mathbf{r}^{\prime}\right)
\end{aligned}
$$


where $\rho_{ \pm}(\mathbf{r})$ is the density profile, and $u_{ \pm}(\mathbf{r})$ is the one-body potential of the cations and/or anions. Note that $f_{++}\left(\mathbf{r}, \mathbf{r}^{\prime}\right)=f_{--}\left(\mathbf{r}, \mathbf{r}^{\prime}\right)$ is the Mayer $f$-function between two like ions, and $f_{+-}\left(\mathbf{r}, \mathbf{r}^{\prime}\right)$ is the Mayer $f$-function between two unlike ions.

The variation of the optimal splitting parameter with the number of cation-anion pairs in the dielectric sphere is given in Fig. 12. The parameter $\sigma$ divides the short-ranged fluctuations from the long-ranged fluctuations, where ion-ion and ion-fixed charge interactions on a length scale less than $\sigma$ are treated within the virial approximation and interactions on a scale longer than $\sigma$ are treated by coupling to the long-range portion of the electrostatic potential. As in the previous cases, the splitting parameter decreases as the number of ions within the confining sphere increases. The increased density dampens fluctuations, which become limited to shorter length scales.

The ion density profiles for $N=5$ ion pairs and $N=100$ ion pairs confined in a sphere with $\eta=1$ are presented in Fig. 13. Due to the symmetry of these systems, the anions and cations have identical density profiles. The MC simulation results show that the ion density is essentially uniform in the sphere for $N=5$ ion pairs with a slight depletion or accumulation (depending on radius of confining sphere) of ions near the surface of the confining sphere. With $N=100$ ion pairs, density maxima appear at the surface for both conditions, the height of the maximum increasing with decreasing radius of the confining sphere. The splitting theory predicts that the ion density profiles have a maximum at the center of the sphere. We attribute this to the cohesive influence of the Coulomb interaction. The absence of the density maxima at the spherical surface comes from the poor treatment of the short-range repulsion between the ions.

Figure 14 shows the ion density profiles at $\eta=10$, where again the surface polarization causes the ions to be repelled from the surface of the confining sphere. As compared to Fig. 13, we have a lower ion density near the surface of the sphere, otherwise the dependence on (i) $R / l_{B}$ and (ii) $N$ remain. The splitting theory is not able to fully describe the reduced ion density near the surface of the sphere. Consequently, the ion density becomes too high in the center of the sphere. As with the other systems discussed previously, the theory performs worst for the case $\eta=10$.

Finally, we present the ion density profiles for $\eta=0.1$ in Fig. 15. In all cases, density maxima appear near the surface due to the polarization of the dielectric interface, which attracts the ions to the surface of the confining sphere. The theory is able to reasonably reproduce the MC simulation results with the exception of the highest density system, where the poor description of the excluded volume effects becomes obvious. Hence, as long as the excluded volume interactions do not dominate the structure of the system, e.g., when the radius of the sphere is not too small, the splitting theory appears to perform well. This holds in particular for the system with $N=100$. 


\section{CONCLUSIONS}

In this work, we have examined systems of ions confined within a dielectric sphere. Three types of systems were examined. The first was a set of identical ions where the charge was neutralized by a uniform surface charge distributed on the surface of the sphere. The next was for identical ions neutralized by a uniform volume charge density throughout the confining sphere. The final system was ions and counterions confined within a dielectric sphere.

For the systems with a uniform surface charge, the splitting theory is able to predict the ion density profiles fairly well for the case $\eta \leq 1$, in comparison with the Monte Carlo simulation results. The virial correction improves the predictions and becomes more important as $\eta$ decreases and as the ions become more confined. In the case of a uniform volume charge, the predictions of the splitting theory worsen, although they are still reasonable for $\eta \leq 1$. The virial correction is much more significant for these systems. When the dielectric constant of the cavity was higher than that of the outside medium, i.e., $\eta>1$, the splitting theory performed poorly both for surface and volume charge distributions. Interestingly, it was found that setting the splitting parameter $\sigma=0$, which corresponds treating the ion image-charge interactions as a one-body potential and the charge-charge interactions within a mean-field approximation, leads to improved predictions; however, the results become increasingly poorer when the confinement becomes significant. Finally, when both ions and counterions are confined within the spherical dielectric cavity, the splitting theory provides reasonable predictions for the ion density profiles, as long as the excluded-volume interactions between the ions do not dominate the structure.

Note that in the systems that we have studied here, the ions are strongly confined, with respect to the strength of their electrostatic interactions. This has provided a severe test of the splitting theory. From this work, it appears that for these confined systems the short-wavelength fluctuations of the ions make a significant contribution in determining the properties of these systems. These fluctuations become especially important when the dielectric constant of the outside medium is lower than that inside the cavity. While the strong-coupling expansion has been successful in describing the systems with high charge densities, it relies on the counterions to be largely separated from each other; however, in the systems we examined in this work, the confining sphere forces the ions to be close to one another. This is most likely the cause of the breakdown of the splitting theory. Therefore, another approximation scheme is required to treat the short-wavelength fluctuations for these systems. We plan to pursue this in future work. 


\section{REFERENCES}

${ }^{1}$ R. D. Coalson and A. Duncan, J. Chem. Phys. 97, 5653 (1992).

${ }^{2}$ R. R. Netz and H. Orland, Eur. Phys. J. E 1, 203 (2000).

${ }^{3}$ R. A. Curtis and L. Lue, J. Chem. Phys. 123, 174702 (2005).

${ }^{4}$ M. M. Hatlo, R. A. Curtis, and L. Lue, J. Chem. Phys. 128, 164717 (2008).

${ }^{5}$ S. Buyukdagli, M. Manghi, and J. Palmeri, Phys. Rev. E 81, 041601 (2010).

${ }^{6}$ S. Buyukdagli, M. Manghi, and J. Palmeri, J. Chem. Phys. 134, 074706 (2011).

${ }^{7}$ S. Buyukdagli, C. V. Achim, and T. Ala-Nissila, J. Chem. Phys. 137, 104902 (2012).

${ }^{8}$ D. S. Dean and R. R. Horgan, Phys. Rev. E 69, 061603 (2004).

${ }^{9}$ D. S. Dean and R. R. Horgan, Phys. Rev. E 70, 011101 (2004).

${ }^{10}$ S. Buyukdagli and T. Ala-Nissila, J. Chem. Phys. 140, 064701 (2014).

${ }^{11}$ B. I. Shklovskii, Phys. Rev. E 60, 5802 (1999).

${ }^{12}$ A. G. Moreira and R. R. Netz, Phys. Rev. Lett. 87, 078301 (2001).

${ }^{13}$ A. Naji, M. Kanduč, J. Forsman, and R. Podgornik, J. Chem. Phys. 139, 150901 (2013).

${ }^{14}$ L. Šamaj and E. Trizac, Phys. Rev. Lett. 106, 078301 (2011).

${ }^{15}$ Y.-G. Chen, C. Kaur, and J. Weeks, J. Phys. Chem. B 108, 19874 (2004).

${ }^{16}$ Y.-G. Chen and J. D. Weeks, Proc. Nat. Acad. Sci. (USA) 103, 7560 (2006).

${ }^{17}$ C. D. Santangelo, Phys. Rev. E 73, 041512 (2006).

${ }^{18}$ M. M. Hatlo and L. Lue, Soft Matter 5, 125 (2009).

${ }^{19}$ M. M. Hatlo and L. Lue, EPL (Europhysics Letters) 89, 25002 (2010).

${ }^{20}$ L. Lue and P. Linse, J. Chem. Phys. 135, 224508 (2011).

${ }^{21}$ P. Linse and L. Lue, J. Chem. Phys. 140, 044903 (2014).

${ }^{22}$ J. M. Rodgers, C. Kaur, Y.-G. Chen, and J. D. Weeks, Phys. Rev. Lett. 97, 097801 (2006).

${ }^{23}$ N. A. Denesyuk and J. D. Weeks, J. Chem. Phys. 128, 124109 (2008).

${ }^{24}$ M. M. Hatlo, P. Banerjee, J. Forsman, and L. Lue, J. Chem. Phys. 137, 064115 (2012).

${ }^{25}$ A.G. Moreira and R.R. Netz, Eur. Phys. J. E 8, 33 (2002).

${ }^{26}$ J. P. Mallarino, G. Téllez, and E. Trizac, J. Phys. Chem. B 117, 12702 (2013).

${ }^{27}$ P. Linse, MOLSIM, Version 5.0, Lund University, Sweden (2011).

${ }^{28}$ A. Bakhshandeh, A. P. dos Santos, and Y. Levin, Phys. Rev. Lett. 107, 107801 (2011).

${ }^{29}$ A. Diehl, A. P. dos Santos, and Y. Levin, J. Phys.: Condensed Matter 24, 284115 (2012). 

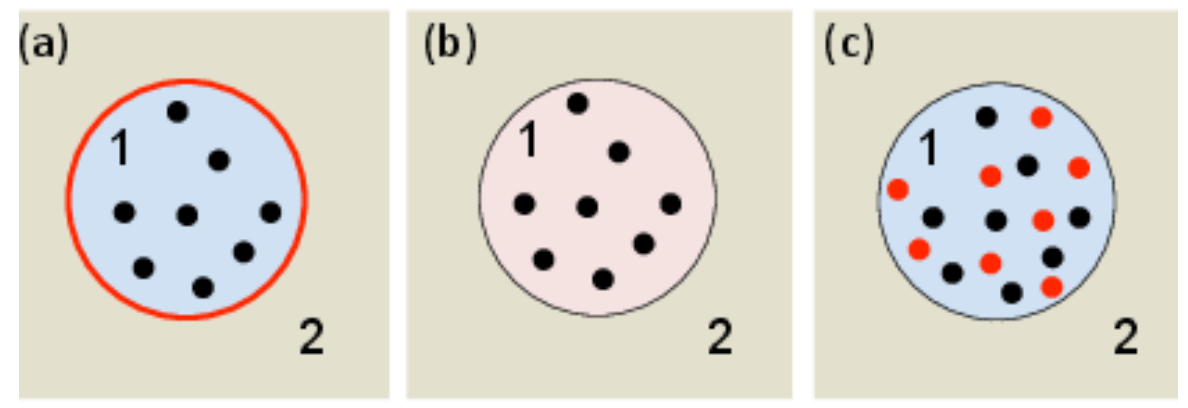

FIG. 1. Overview of different types of systems. Systems of all types contain a spherical region of medium 1 with dielectric constant $\varepsilon_{1}$ embedded in medium 2 of infinite size with dielectric constant $\varepsilon_{2}$. Moreover, in (a) region 1 is surrounded by a uniform surface charge density and contains only counterions, in (b) region 1 possesses a uniform volume charge density and contains only counterions, and in (c) both co- and counterions are present, but no uniform charge density. In all type of systems, the mobil ions are confined within the sphere. All the systems are electrically neutral. 


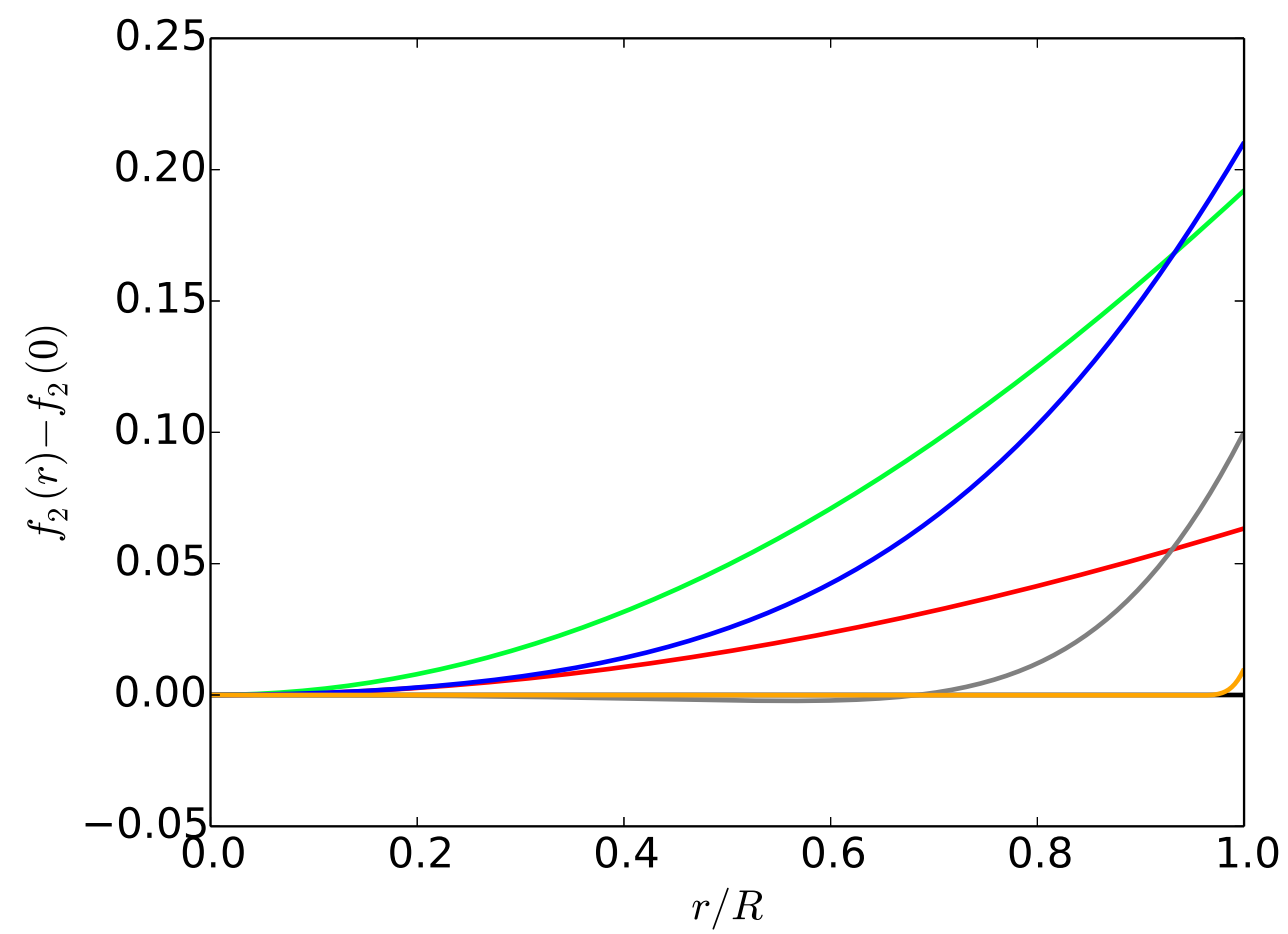

FIG. 2. Effective short-wavelength electrostatic potential generated by a uniform surface charge density as a function of the radial position for $\sigma / R \rightarrow \infty$ (black), $\sigma / R=1$ (red), $\sigma / R=0.5$ (green), $\sigma / R=0.2$ (blue), $\sigma / R=0.1$ (gray), and $\sigma / R=0.01$ (orange) 


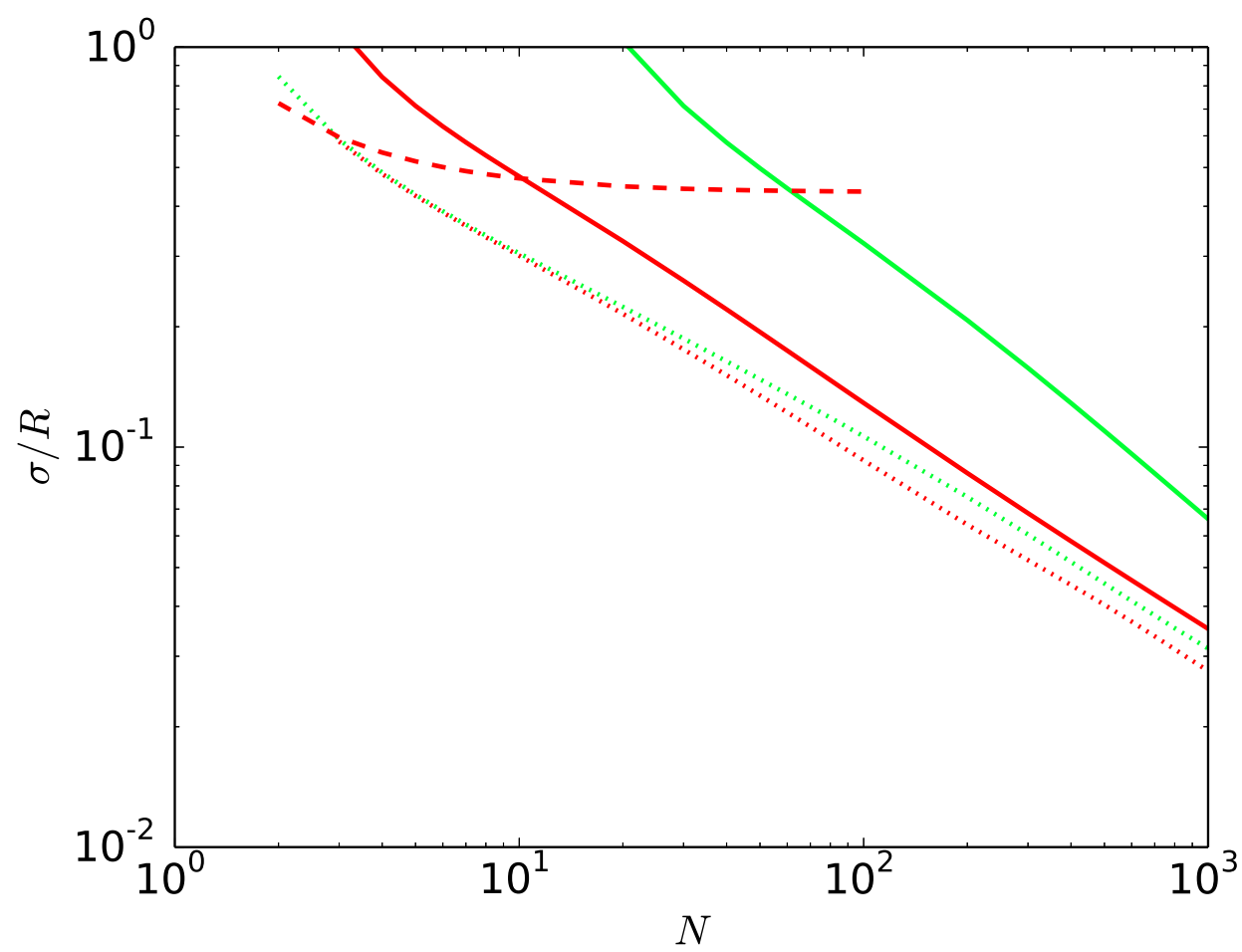

FIG. 3. Optimal reduced splitting parameter $\sigma / R$ for the splitting theory with the virial correction as a function of the number of counterions $N$ confined in a spherical cavity with a uniform surface charge density and radius $R / l_{B}=1$ (red) and $R / l_{B}=10$ (green) for $\eta=1$ (solid lines), $\eta=10$ (dashed lines), and $\eta=0.1$ (dotted lines). In the case $\eta=10$ and $R / l_{B}=10, \sigma / R \rightarrow \infty$. 


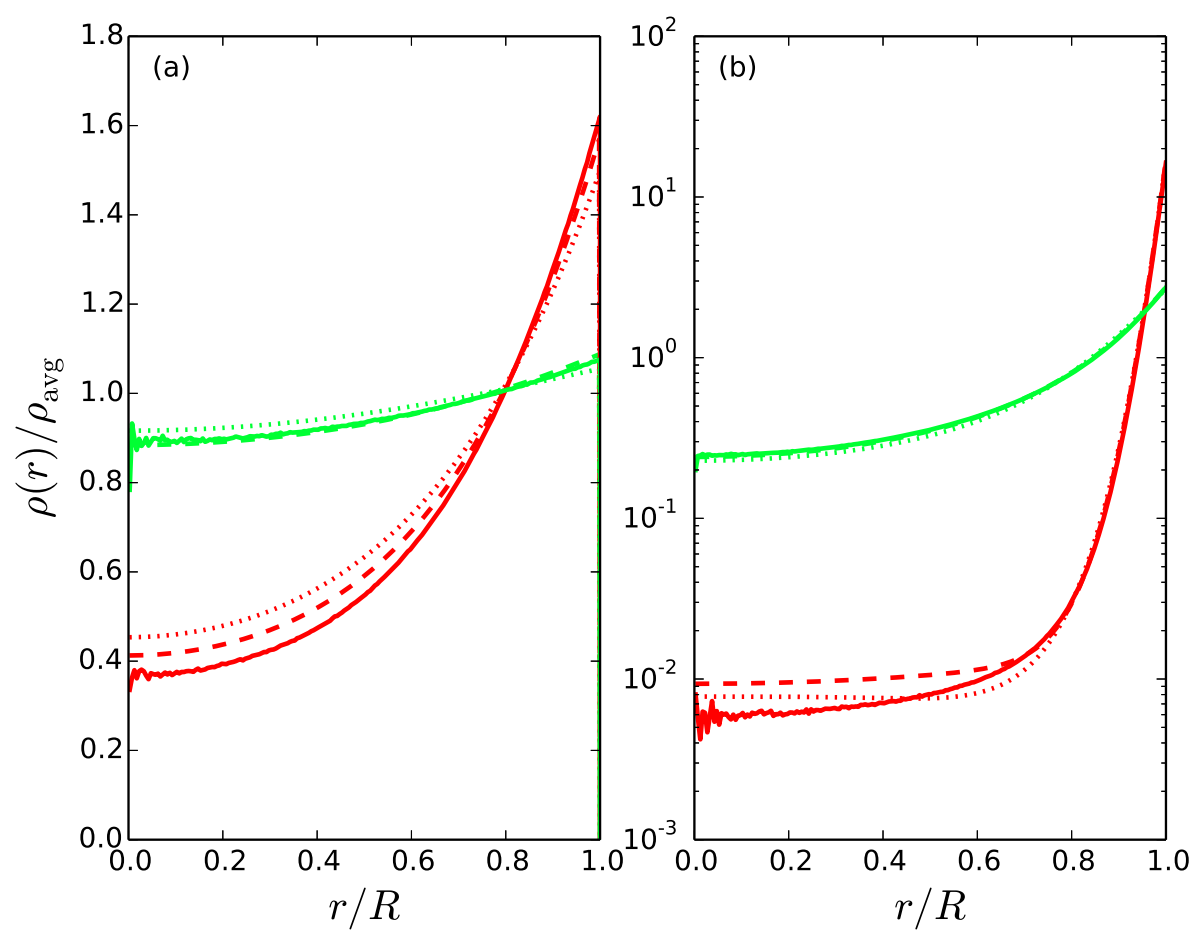

FIG. 4. Counterion density profiles for (a) $N=5$ and (b) $N=100$ counterions within a spherical cavity with a uniform surface charge density and $\eta=1$ for $R / l_{B}=1$ (red) and $R / l_{B}=10$ (green) predicted from Monte Carlo simulations (solid lines), the splitting theory (dotted lines), and the splitting theory with the virial correction (dashed lines). 


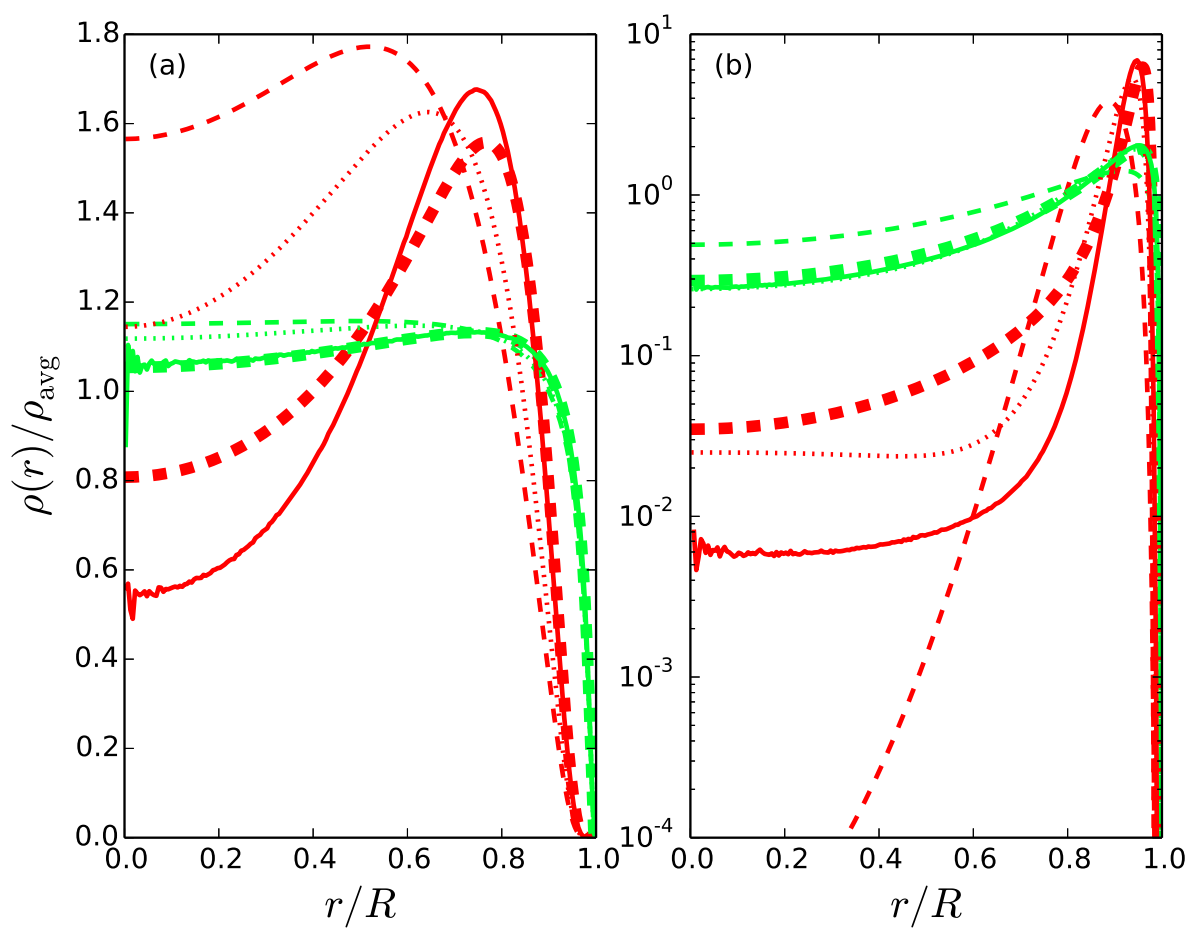

FIG. 5. Counterion density profiles for (a) $N=5$ and (b) $N=100$ counterions within a spherical cavity with a uniform surface charge density and $\eta=10$, where lines have the same meaning as in Fig. 4. Predictions of the spitting theory with the virial correction and $\sigma=0$ are also included (thick dashed lines). 


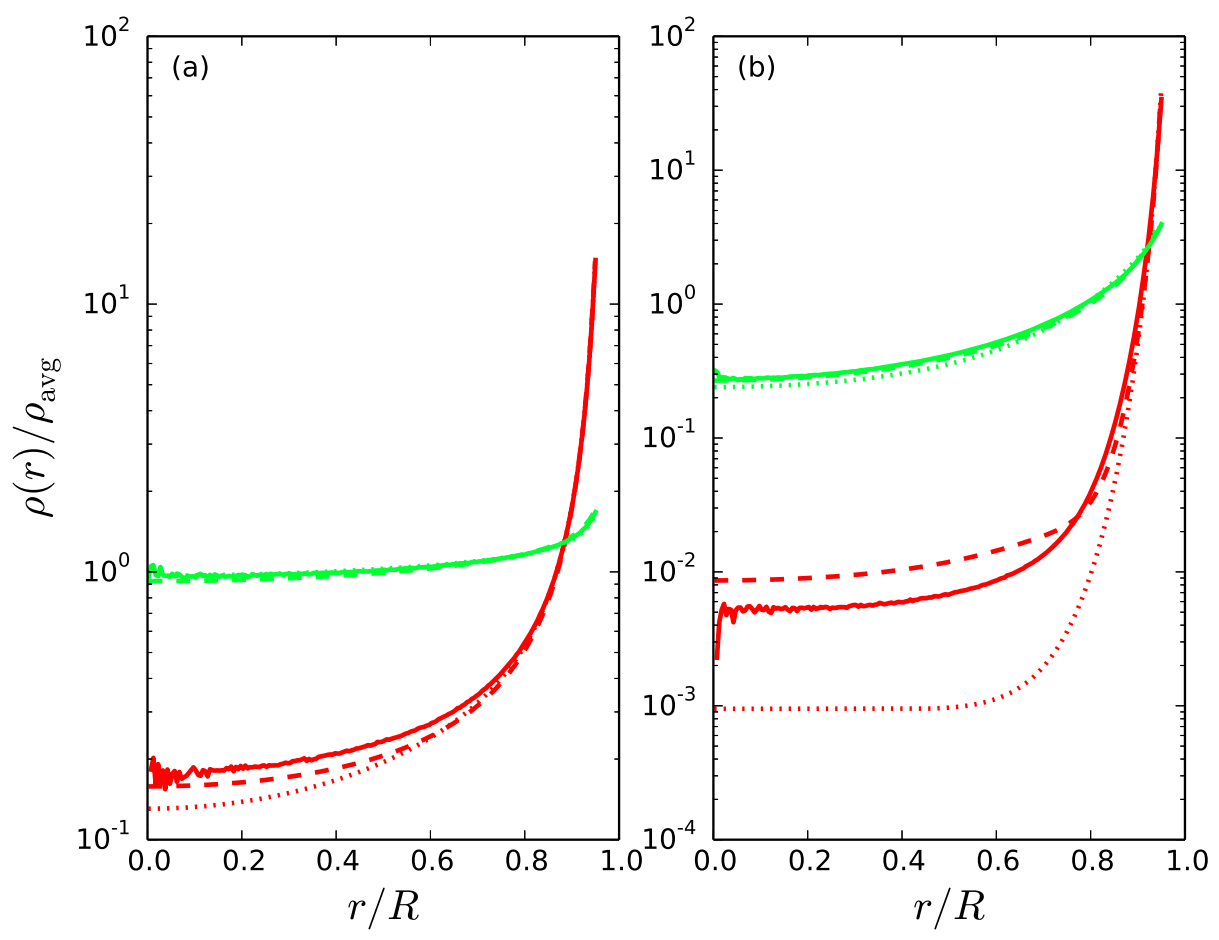

FIG. 6. Counterion density profiles for (a) $N=5$ and (b) $N=100$ counterions within a spherical cavity with a uniform surface charge density and $\eta=0.1$, where lines have the same meaning as in Fig. 4. 


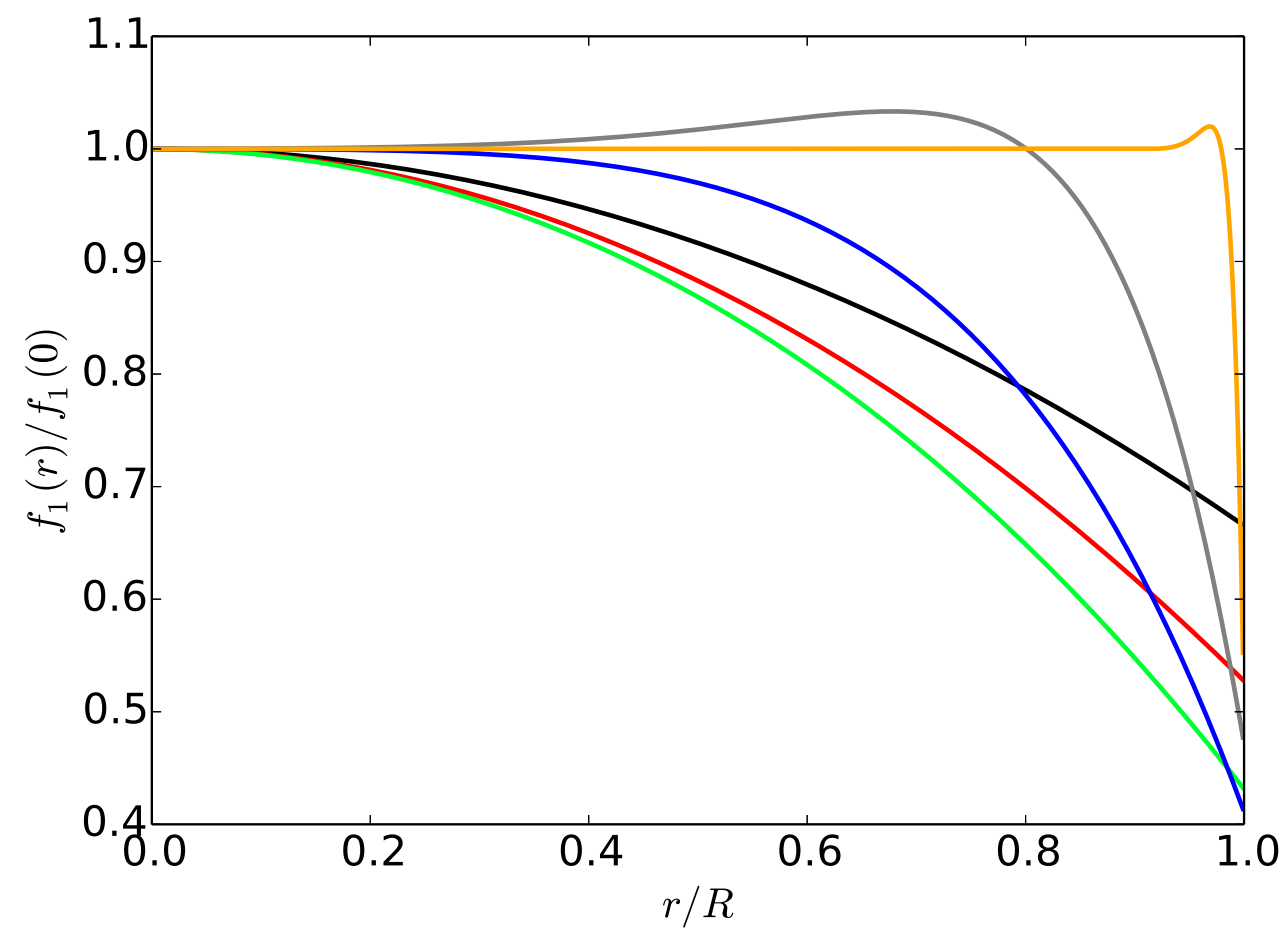

FIG. 7. Effective short-wavelength electrostatic potential generated by a uniform volume charge density as a function of the radial position for $\sigma / R \rightarrow \infty$ (black), $\sigma / R \rightarrow 1$ (red), $\sigma / R \rightarrow 0.5$ (green), $\sigma / R \rightarrow 0.2$ (blue), $\sigma / R \rightarrow 0.1$ (gray), and $\sigma / R \rightarrow 0.01$ (orange). 


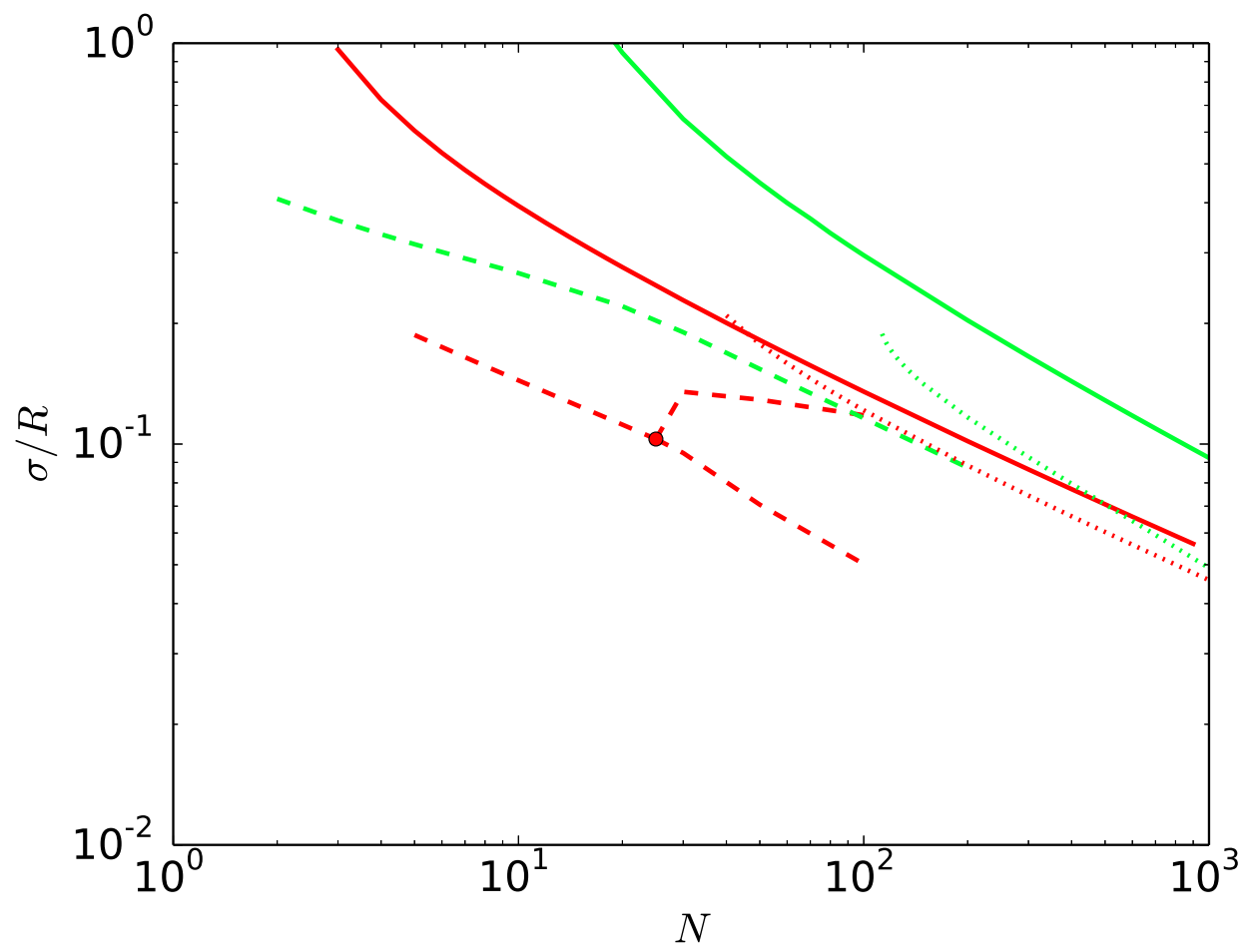

FIG. 8. Optimal reduced splitting parameter $\sigma / R$ for the splitting theory with the virial correction as a function of the number of counterions $N$ confined in a spherical cavity with a uniform volume charge density and radius $R / l_{B}=1$ (red) and $R / l_{B}=10$ (green) for $\eta=1$ (solid lines), $\eta=10$ (dashed lines), and $\eta=0.1$ (dotted lines). 

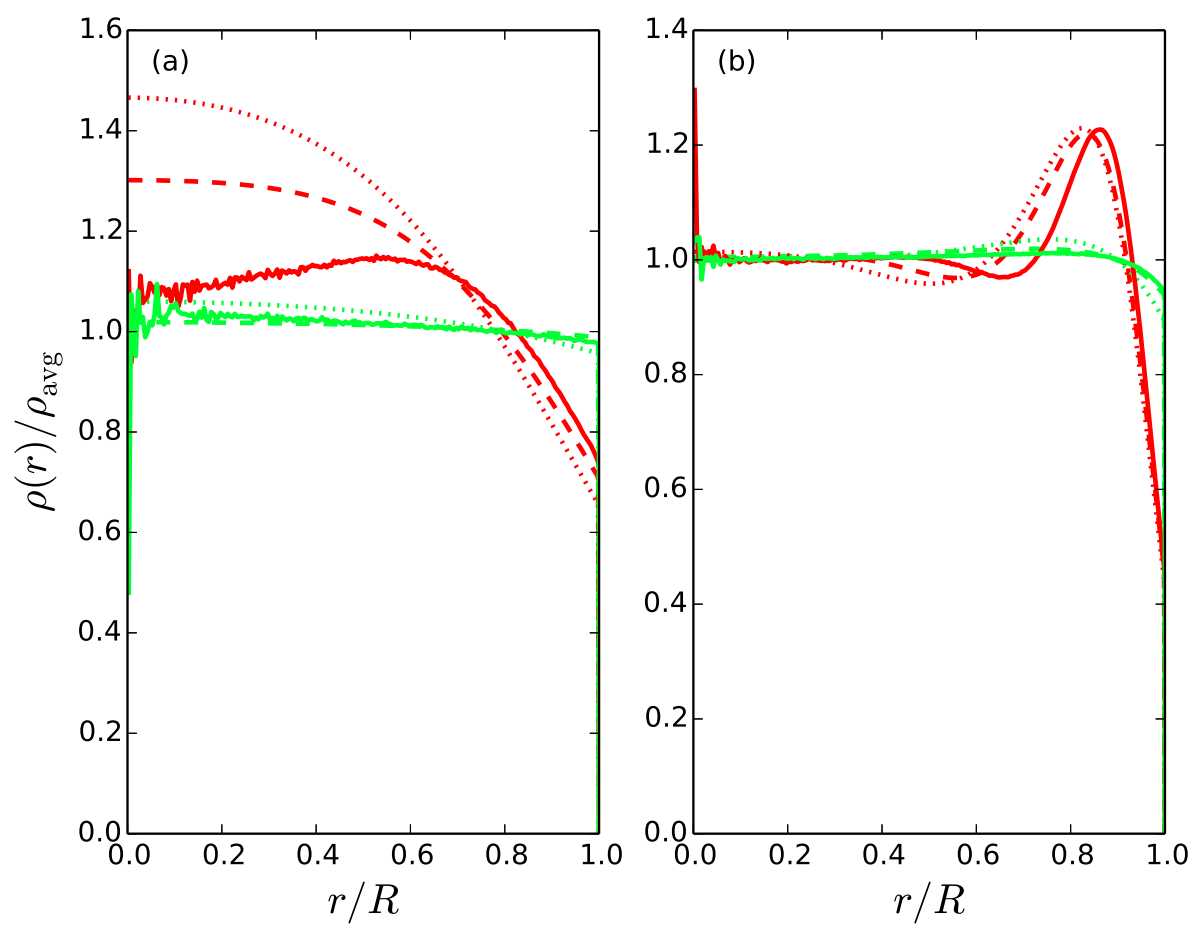

FIG. 9. Counterion density profiles for (a) $N=5$ and (b) $N=100$ counterions within a spherical cavity with a uniform volume charge density and $\eta=1$, where lines have the same meaning as in Fig. 4. 

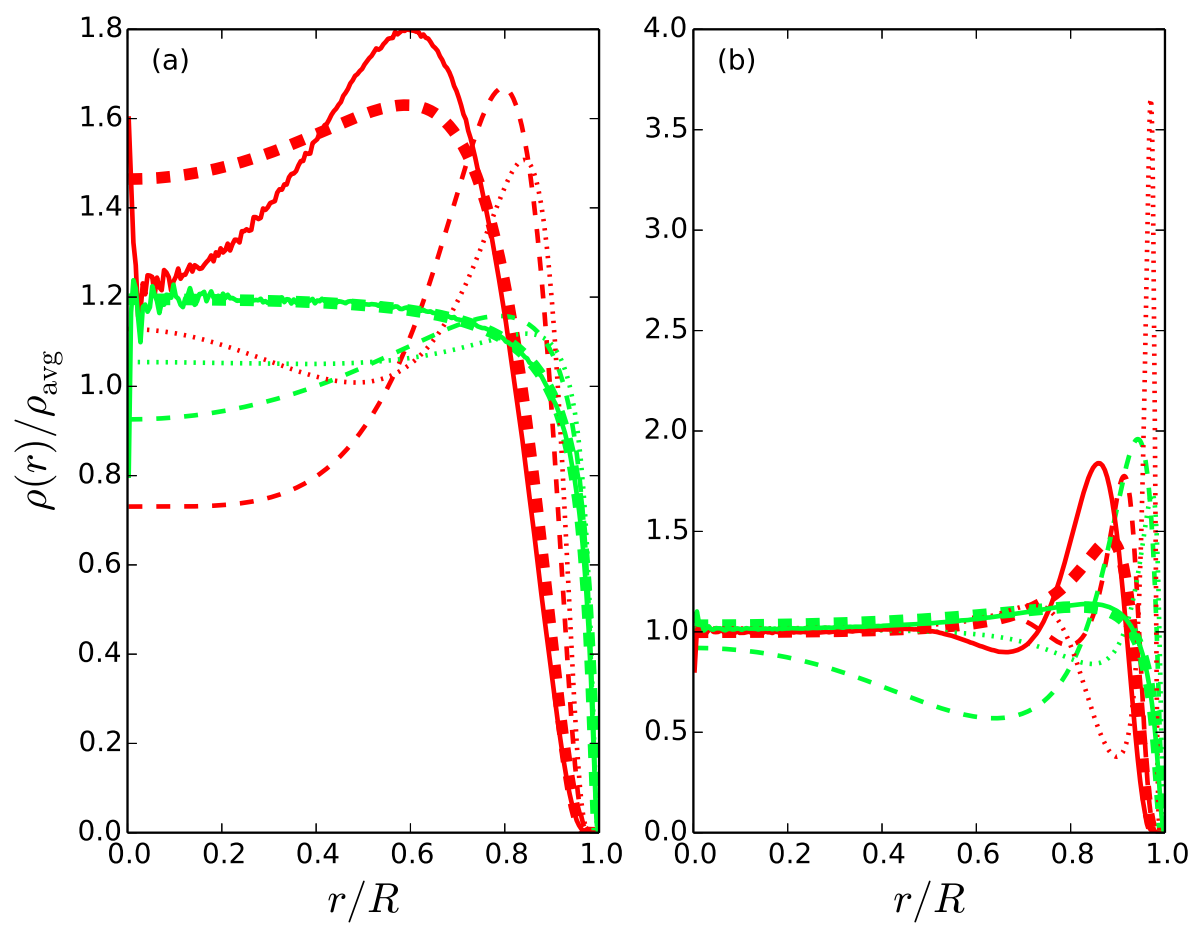

FIG. 10. Counterion density profiles for (a) $N=5$ and (b) $N=100$ counterions within a spherical cavity with a uniform volume charge density and $\eta=10$, where lines have the same meaning as in Fig. 4. Predictions of the spitting theory with the virial correction and $\sigma=0$ are also included (thick dotted lines). 

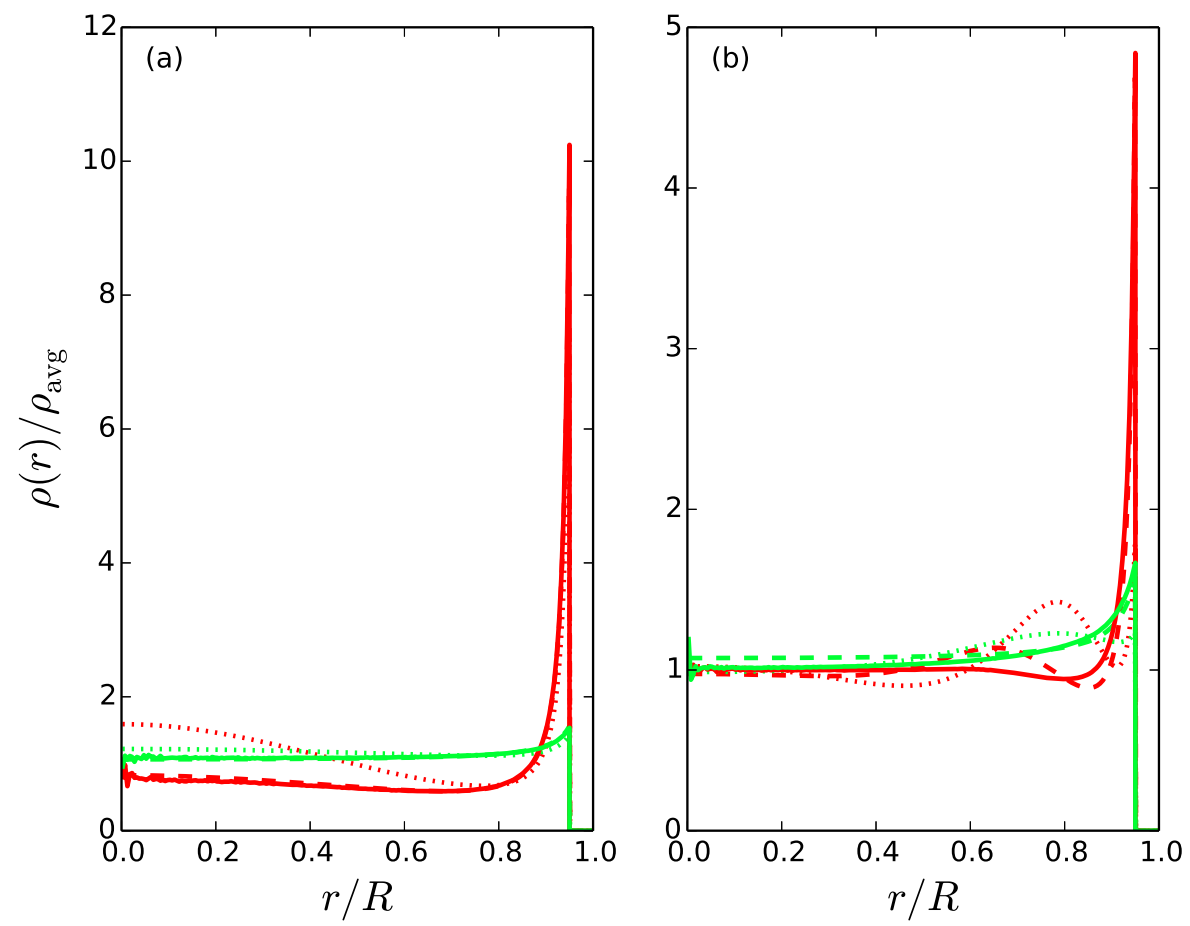

FIG. 11. Counterion density profiles for (a) $N=5$ and (b) $N=100$ counterions within a spherical cavity with a uniform volume charge density and $\eta=0.1$, where lines have the same meaning as in Fig. 4. 


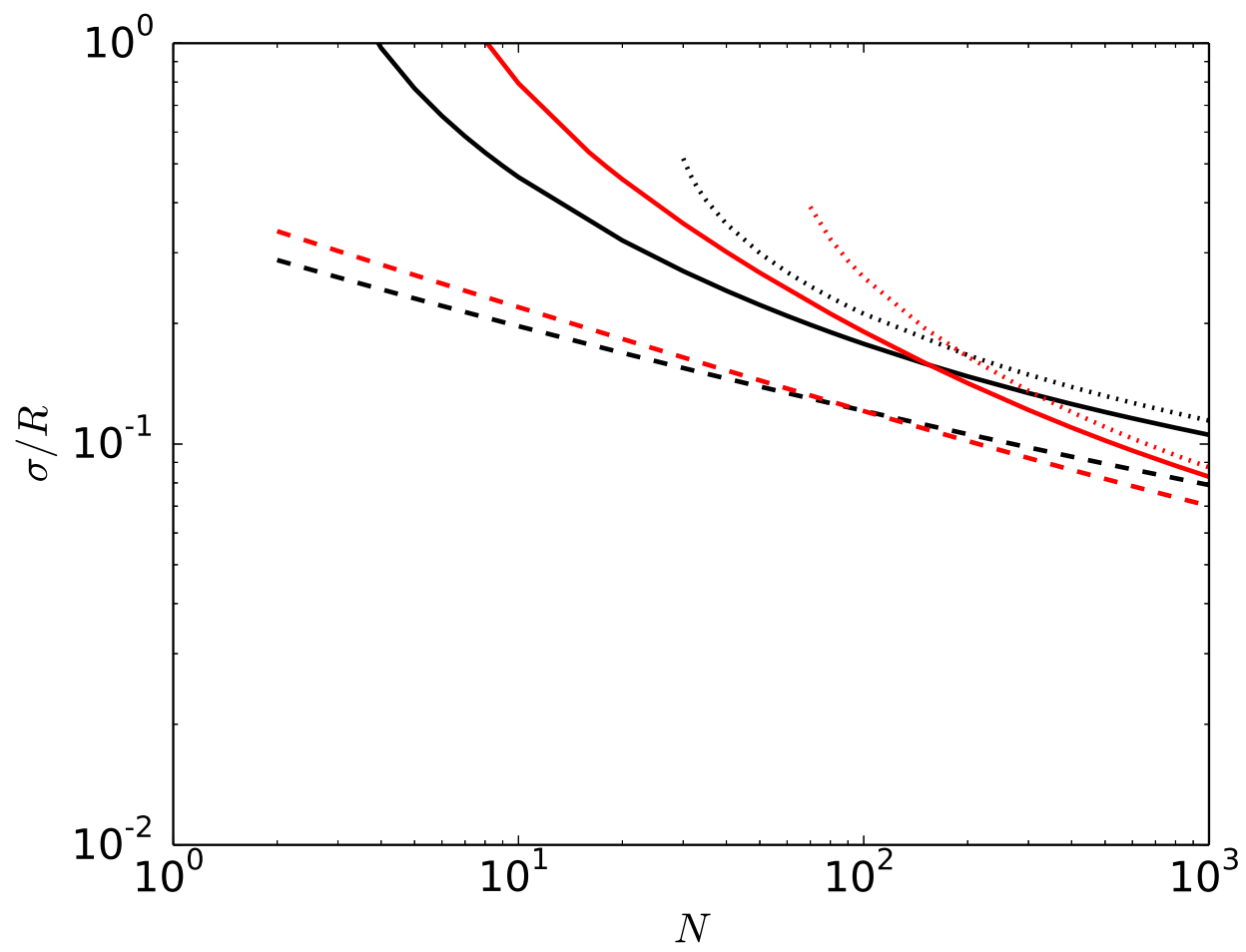

FIG. 12. Optimal reduced splitting parameter $\sigma / R$ for the splitting theory with the virial correction as a function of $N$, where $N$ cations and $N$ anions with hard-sphere radius $R_{\text {ion }}=0.5 l_{B}$ are confined in a spherical cavity with a radius of $R / l_{B}=5$ (black) and $R / l_{B}=10$ (red) for $\eta=1$ (solid lines), $\eta=10$ (dashed lines), and $\eta=0.1$ (dotted lines). 

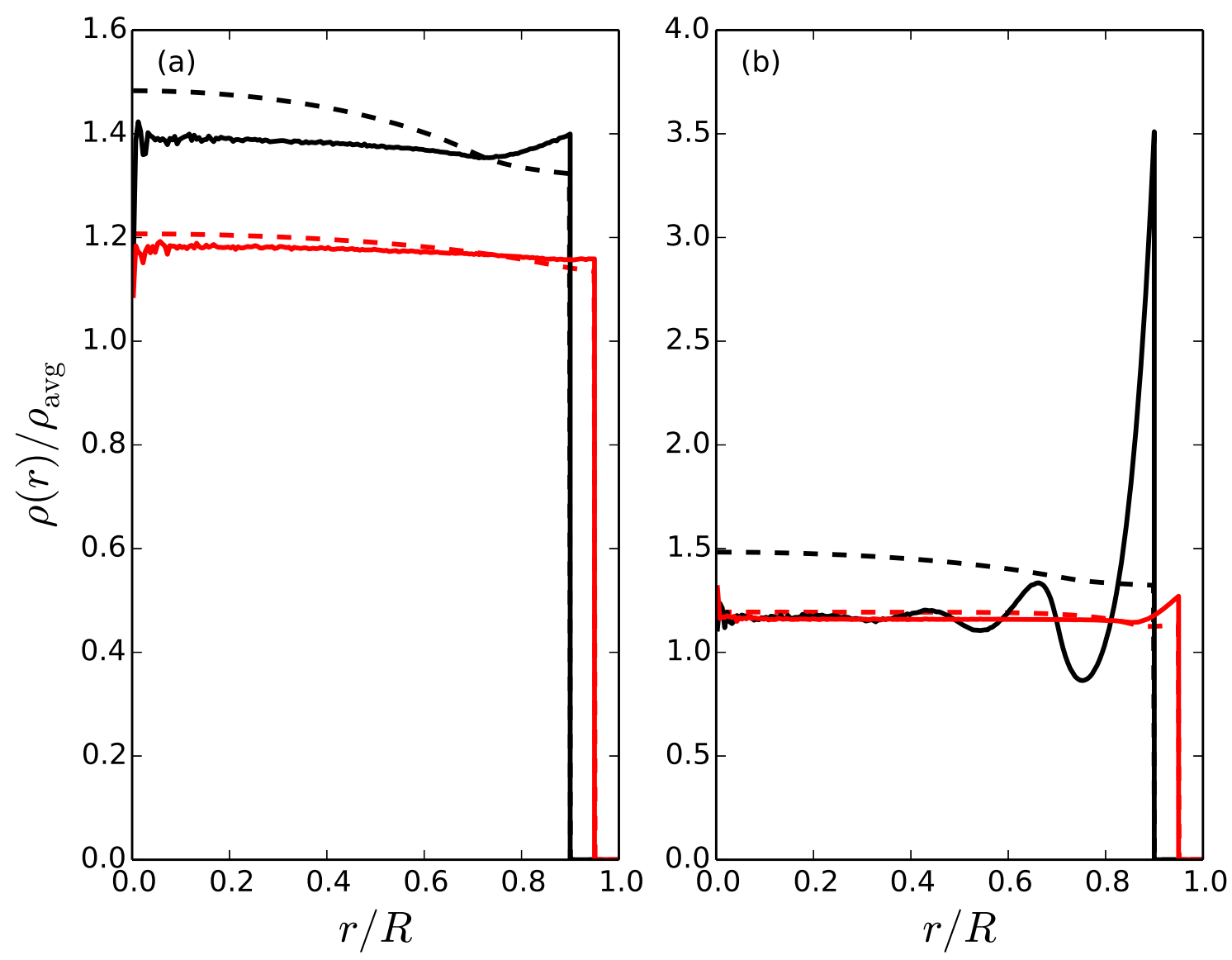

FIG. 13. Ion density profiles for (a) $N=5$ and (b) $N=100$ cations/anions within a spherical cavity with $\eta=1$ and $R / l_{B}=5$ (black) and $R / l_{B}=10$ (red) predicted from Monte Carlo simulations (solid lines) and the splitting theory with the virial correction (dashed lines). 

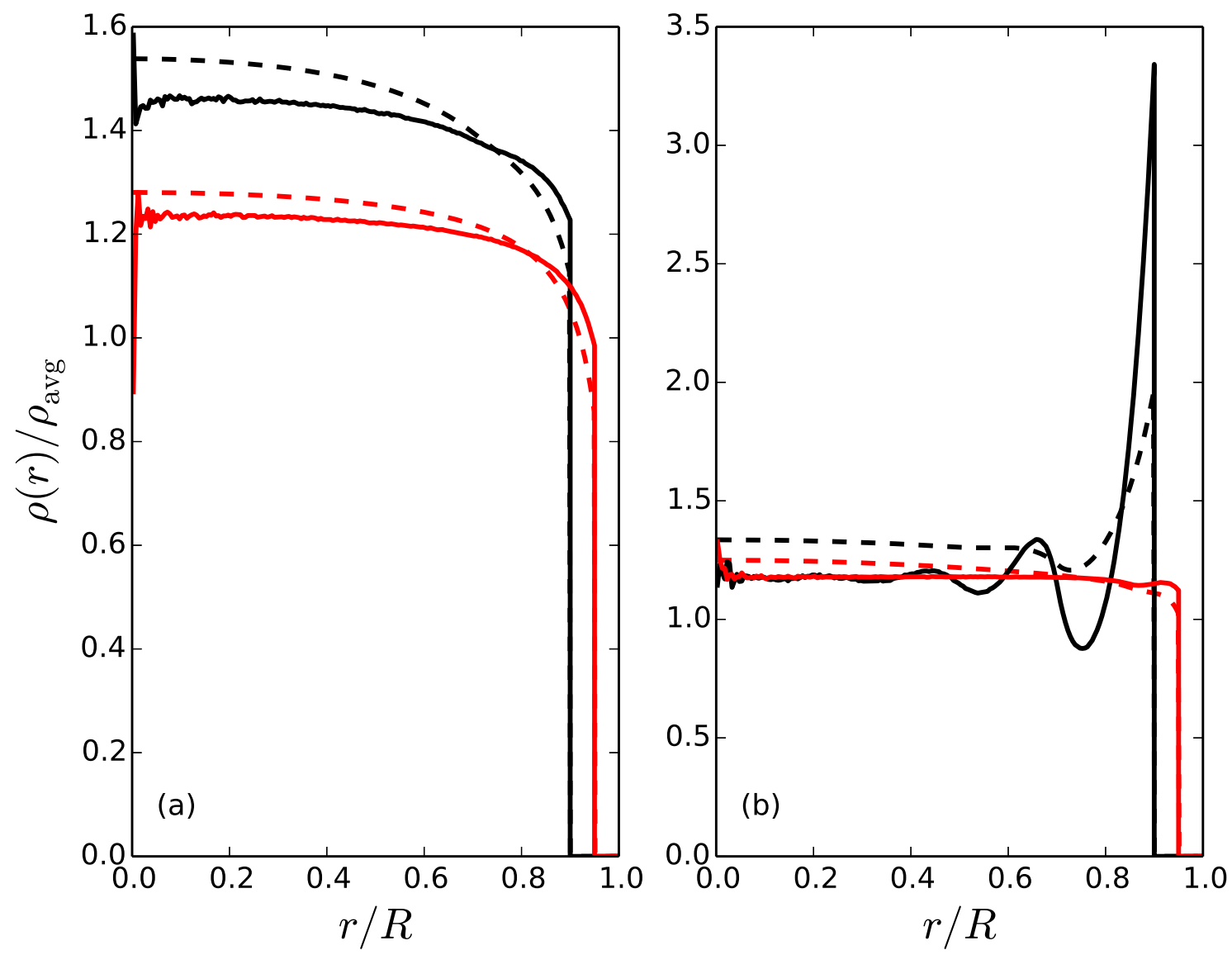

FIG. 14. Ion density profiles for (a) $N=5$ and (b) $N=100$ cations/anions within a spherical cavity with $\eta=10$, where lines have the same meaning as in Fig. 13. 

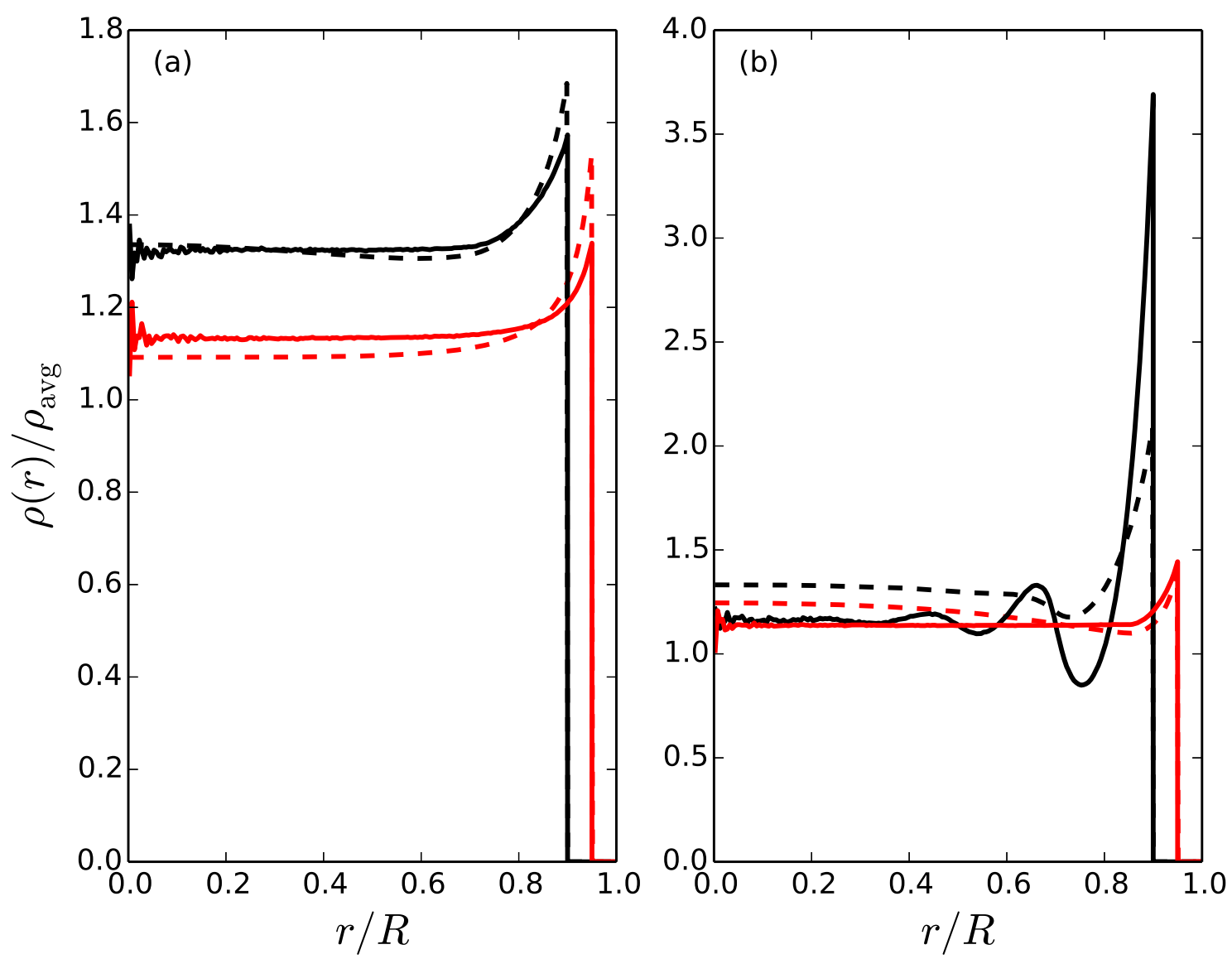

FIG. 15. Ion density profiles for (a) $N=5$ and (b) $N=100$ cations/anions within a spherical cavity with $\eta=0.1$, where lines have the same meaning as in Fig. 13. 